\title{
Géométries Lorentziennes de dimension 3 : classification et complétude
}

\author{
Sorin Dumitrescu • Abdelghani Zeghib
}

Received: 19 October 2009 / Accepted: 15 February 2010 / Published online: 5 March 2010

(C) Springer Science+Business Media B.V. 2010

\begin{abstract}
We classify three-dimensional Lorentz homogeneous spaces $G / I$ having a compact manifold locally modeled on them. We prove a completeness result: any compact locally homogeneous Lorentz threefold $M$ is isometric to a quotient of a Lorentz homogeneous space $G / I$ by a discrete subgroup $\Gamma$ of $G$ acting properly and freely on $G / I$. Moreover, if $I$ is noncompact, $G / I$ is isometric to a Lie group $L$ endowed with a left invariant Lorentz metric, where $L$ is isomorphic to one of the following Lie groups:
\end{abstract}

$$
\mathbf{R}^{3}, S \widetilde{L(2, \mathbf{R})}, \text { Heis or } S O L \text {. }
$$

If $L$ is not $S \widetilde{L(2, \mathbf{R})}$, then $M$ admits a finite cover which is a quotient of $L$ by a lattice.

Keywords Locally homogenous Lorentz manifolds - Transitive Killing Lie algebras . Completeness of $(G, X)$-structures

\section{Mathematics Subject Classification (2000) $\quad 53 \mathrm{~B} 30 \cdot 53 \mathrm{C} 22 \cdot 53 \mathrm{C} 50$}

Résumé Nous classifions les géométries lorentziennes de dimension trois qui admettent des réalisations compactes. Nous démontrons un résultat de complétude : toute variété lorentzienne localement homogène compacte de dimension trois est isométrique au quotient (à gauche) d'un espace homogène lorentzien $G / I$ de dimension trois par un sous-groupe discret $\Gamma$ de $G$ agissant proprement et librement. Si, de plus, le groupe d'isotropie locale de la métrique lorentzienne est supposé non compact, le revêtement universel de la variété est isométrique à un groupe de Lie $L$ muni d'une métrique lorentzienne invariante par translation

S. Dumitrescu $(\varangle)$

Département de Mathématique d'Orsay, Équipe de Topologie et Dynamique, Bat. 425, U.M.R. 8628

C.N.R.S., Univ. Paris-Sud (11), 91405 Orsay Cedex, France

e-mail: Sorin.Dumitrescu@math.u-psud.fr

A. Zeghib

CNRS, UMPA, École Normale Supérieure de Lyon, Lyon Cedex, France

e-mail: zeghib@umpa.ens-lyon.fr 
à gauche et $L$ est isomorphe à l'un des groupes suivants : $\mathbf{R}^{3}, \widetilde{S(2, \mathbf{R})}$, Heis ou $S O L$. Si $L$ n'est pas $S \widetilde{L(2, \mathbf{R})}$, alors la variété admet un revêtement fini qui est isométrique à un quotient de $L$ par un réseau.

Motsclés Variétés lorentziennes localement homogènes · Algèbres de Killing transitives · Complétude géodésique · Complétude des $(G, X)$-structures

\section{Introduction et exemples}

Soit $G$ un groupe de Lie réel et $G / I$, où $I$ est un sous-groupe fermé de $G$, un espace homogène supposé simplement connexe.

On dit que $G / I$ est lorentzien et que $(G, G / I)$ est une géométrie lorentzienne (au sens de Klein) si l'action canonique de $G \operatorname{sur} G / I$ préserve une métrique lorentzienne [i.e. un champ lisse de formes quadratiques non dégénérées de signature $(n-1,1)]$, ou de manière équivalente, si l'action adjointe de $I$ préserve une forme quadratique non dégénérée de signature $(n-1,1)$ sur le quotient $\mathcal{G} / \mathcal{I}$ des algèbres de Lie correspondantes.

Une variété compacte connexe $M$ admet une $(G, G / I)$-structure (on dit aussi que $M$ est localement modelée sur l'espace homogène $G / I)$ s'il existe un atlas de $M$ à valeurs dans des ouverts de $G / I$ tel que les applications de changements de cartes soient données par des éléments du groupe $G$. Dans ce cas, tout objet géométrique (par exemple, un tenseur ou une connexion) sur $G / I$, invariant par l'action de $G$, induit un objet géométrique du même type sur $M$. En particulier, dans le cas d'un espace homogène de type lorentzien $G / I$, la variété $M$ hérite d'une métrique lorentzienne localement homogène (voir la définition en Sect. 3). Nous dirons aussi que la géométrie lorentzienne $(G, G / I)$ admet une réalisation compacte sur la variété $M$.

La géométrie lorentzienne ( $G, G / I)$ est dite maximale, si l'action de $G$ ne s'étend pas en une action fidèle d' un groupe de Lie de dimension strictement plus grande $G^{\prime}$ qui préserve une métrique lorentzienne. Deux métriques lorentziennes sur $G / I$ dont les composantes neutres des groupes des isométries sont conjuguées dans le groupe des difféomorphismes de $G / I$ définissent une même géométrie.

Une $(G, G / I)$-structure sur $M$ donne classiquement naissance à une application développante qui est un difféomorphisme local entre un revêtement universel $\widetilde{M}$ de $M$ et l'espace modèle $G / I$ [37-39] et à un morphisme de groupe $\rho: \pi_{1}(M) \rightarrow G$ qui envoie le groupe fondamental $\pi_{1}(M)$ de $M$ dans $G$ et qui est bien défini à conjugaison près. L'application développante conjugue l'action du groupe de revêtement $\widetilde{M} \rightarrow M$ à l'action du groupe d'holonomie $\Gamma=\rho\left(\pi_{1}(M)\right)$ sur l'espace modèle.

Une $(G, G / I)$-structure est dite complète si son application développante est un difféomorphisme. Lorsque $G$ préserve une métrique lorentzienne ou riemannienne, ou plus généralement une connexion, on a aussi la notion de complétude géodésique [40]. La complétude géodésique est plus forte que la complétude au sens ci-dessus (voir lemme 2.2).

Si $M$ est complète, alors $M=\Gamma \backslash G / I$, où $\Gamma$ est le groupe d'holonomie qui agit librement et proprement sur $G / I$. Lorsque $I$ est compact, ceci équivaut au fait que $\Gamma$ soit un réseau cocompact de $G$.

On dit qu'une $(G, G / I)$-structure satisfait à une rigidité de Bieberbach, si pour toute réalisation compacte complète $M$, il existe un sous-groupe de Lie connexe $L$ de $G$ contenant, à indice fini près, le groupe d'holonomie $\Gamma$ de $M$ et agissant librement et transitivement sur $G / I$. Dans ce cas, $L$ s'identifie à $G / I$ et, à indice fini près, $M$ est $\Gamma \backslash L$. L'énoncé classique 
du théorème de Bieberbach correspond au cas de la géométrie euclidienne $\left(O(n) \ltimes \mathbf{R}^{n}, \mathbf{R}^{n}\right)$, avec $L=\mathbf{R}^{n}$, le groupe des translations.

Rappelons que W. Thurston a classifié les huit géométries riemanniennes maximales de dimension trois qui possèdent des réalisations compactes (voir [37-39]).

Une spécificité bien connue des $(G, G / I)$-structures riemanniennes est que toute réalisation compacte d'une telle structure est nécessairement complète. Ce phénomène n'est nullement assuré (et souvent faux) pour les $(G, G / I)$ )-structures générales, mais sera démontré dans cet article pour les $(G, G / I)$-structures lorentziennes de dimension 3.

Nous nous intéressons dans ce travail aux géométries lorentziennes de dimension 3 qui sont non-riemanniennes, i.e. l'action de $G$ sur $G / I$ ne préserve pas de métrique riemannienne. C'est équivalent au fait que l'action adjointe de $I$ soit à image non bornée dans le groupe des transformations linéaires de $\mathcal{G} / \mathcal{I}$.

Dans la suite de cette introduction on explicite des exemples de géométries lorentziennes non-riemanniennes maximales de dimension 3.

Les géométries lorentziennes de courbure sectionnelle constante sont maximales car elles réalisent la dimension maximale du groupe des isométries (voir proposition 3.1 ou [40]).

Avant de présenter les géométries modèles $(G, G / I)$ qui incarnent, en dimension 3 , les géométries lorentziennes de courbure sectionnelle constante et qui sont la géométrie plate de Minkowski (courbure nulle), la géométrie de Sitter (courbure positive) et la géométrie anti de Sitter (courbure négative), précisons que, d'après un résultat dû à Carrière [7] dans le cas plat et étendu par Klingler [22] au cas de courbure sectionnelle constante (voir également $[26,29])$, toute réalisation compacte de $G / I$ est complète. Ce résultat de complétude est essentiel dans l'étude des géométries lorentziennes de courbure sectionnelle constante.

Il implique, en particulier, que la géométrie de courbure sectionnelle constante positive n'admet pas de réalisation compacte. En effet, d'après les travaux [4], seuls les groupes finis agissent proprement sur l'espace de Sitter, qui n'admet donc pas de quotient compact.

Géométrie Minkowski Un modèle de la géométrie Minkowski est $\mathbf{R}^{3}$, muni de la forme quadratique $d x^{2}+d y^{2}-d z^{2}$. Le groupe $G$ de cette géométrie est $O(2,1) \ltimes \mathbf{R}^{3}$, agissant affinement sur $\mathbf{R}^{3}$. L'isotropie $I$ de la géométrie Minkowski est $O(2,1)$.

Il est démontré dans $[13,15]$ que la géométrie Minkowski satisfait à une rigidité de Bieberbach, avec le groupe $L$ isomorphe à $\mathbf{R}^{3}$, Heis ou $S O L$.

Géométrie anti de Sitter Un modèle de cette géométrie est le revêtement universel $\widehat{S L(2, \mathbf{R})}$ de $S L(2, \mathbf{R})$, muni de la métrique lorentzienne invariante par translations à gauche qui coïncide en identité avec la forme de Killing $q$ sur l'algèbre de Lie $s l(2, \mathbf{R})$. Comme la forme de Killing $q$ est invariante par la représentation adjointe, le groupe des isométries de la géométrie anti de Sitter contient également les translations à droite. La composante neutre du groupe des isométries de la géométrie anti de Sitter est $G=S \widetilde{L(2, \mathbf{R})} \times{ }_{Z} \widehat{S L(2, \mathbf{R})}$, où $Z$ est le centre du groupe, avec isotropie $I$ isomorphe à $S \widetilde{L(2, \mathbf{R})}$ et plongée diagonalement dans $G$.

Comme le groupe $S \widetilde{L(2, \mathbf{R})}$ agit librement transitivement sur le modèle $G / I$, il suffit

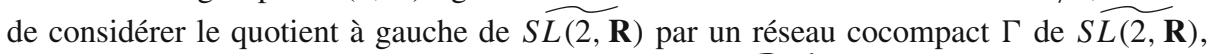
pour construire ainsi des variétés compactes $M=\Gamma \backslash S \widetilde{L(2, \mathbf{R})}$ localement modelées sur la géométrie anti de Sitter.

La rigidité de Bieberbach, valable dans le cas plat, n'est plus valide pour la géométrie anti de Sitter [14,36].

Géométrie Lorentz-Heisenberg Il s'agit de la géométrie d'une certaine métrique lorentzienne invariante par translations à gauche sur le groupe de Heisenberg Heis. Désignons par heis l'algèbre de Lie de Heis. 
Proposition 1.1 Modulo automorphisme et à constante multiplicative près, il existe sur Hei s une seule métrique lorentzienne invariante à gauche affectant une longueur strictement positive au centre de heis. Ces métriques définissent une même géométrie lorentzienne nonriemannienne maximale, dont la composante neutre du groupe des isométries est de dimension quatre, isomorphe à un produit semi-directe $\mathbf{R} \ltimes H$ ei st dont l'isotropie est semi-simple (i.e. agit sur l'espace tangent au point base comme un groupe à un paramètre diagonalisable). Cette géométrie sera appelée Lorentz-Heisenberg.

Comme avant, il suffit de considérer un quotient (à gauche) de Heis par un réseau cocompact $\Gamma$, pour se convaincre que la géométrie Lorentz-Heisenberg se réalise bien sur des variétés compactes.

Géométrie Lorentz-SOL. C'est une géométrie obtenue à partir d'une métrique lorentzienne invariante à gauche sur $S O L$. Rappelons que l'algèbre de Lie correspondante sol est engendrée par $\{X, Z, T\}$, avec seuls crochets non-nuls $[T, X]=X$ et $[T, Z]=-Z$. L'algèbre dérivée est donc $\mathbf{R}^{2}=\mathbf{R} X \oplus \mathbf{R} Z$. Considérons sur $S O L$ la métrique lorentzienne invariante à gauche $g$ définie en identité par : $X$ et $T$ sont isotropes, $Z$ est orthogonal à $\mathbf{R} X \oplus \mathbf{R} T$, et $g(X, T)=g(Z, Z)=1$.

Proposition 1.2 À automorphisme près, g est l'unique métrique lorentzienne invariante à gauche sur SOL, qui rend l'algèbre dérivée dégénérée et telle que l'une des deux directions propres de ad $(T)$ est isotrope. La composante neutre du groupe des isométries de g est de dimension 4 : il s'agit d'une extension non triviale de Heis (ici, Heis n'agit pas transitivement) et l'isotropie locale est non compacte (unipotente). Cette métrique définit une géométrie lorentzienne maximale et non-riemannienne qu'on désignera par Lorentz-SOL.

Les propositions 1.1 et 1.2 seront démontrées à la Sect. 4 .

\section{2 Énoncés des résultats}

Le théorème principal de cet article montre, en particulier, que les exemples précédents constituent les seules géométries lorentziennes non-riemanniennes maximales qui se réalisent sur des variétés compactes de dimension 3 :

Théorème 2.1 Soit M une variété lorentzienne compacte connexe de dimension 3 localement modelée sur une géométrie lorentzienne non-riemannienne $(G, G / I)$.

Classification:

(i) Le modèle G/I est isométrique à une métrique lorentzienne invariante à gauche sur l'un des quatre groupes suivants: $\mathbf{R}^{3}, \widehat{S L(2, \mathbf{R})}$, Heis ou $S O L$.

- Dans le cas de $\mathbf{R}^{3}$, toutes les métriques sont plates.

- Dans le cas de $S \widetilde{L(2, \mathbf{R})}$, il y a trois géométries lorentziennes non-riemanniennes qui proviennent des métriques lorentziennes invariantes à gauche : la seule géométrie maximale est anti de Sitter, les deux autres sont données par des métriques lorentziennes de courbure sectionnelle non constante invariantes à gauche et également par un sous-groupe à un paramètre unipotent (respectivement semisimple) de translations à droite.

- Dans le cas de Heis, il y a deux géométries lorentziennes non-riemanniennes invariantes à gauche. L'une est plate et l'autre correspond à la géométrie LorentzHeisenberg. 
- Dans le cas de SOL, il y a deux géométries lorentziennes non-riemanniennes invariantes à gauche. L'une est plate et l'autre correspond à la géométrie LorentzSOL.

(ii) Si la géométrie lorentzienne ( $G, G / I)$ est maximale, alors c'est l'une des 4 géométries suivantes : Minkowski, anti de Sitter, Lorentz-Heisenberg ou Lorentz-SOL. Complétude:

(iii) La $(G, G / I)$-structure est complète.

(iv) On a une rigidité de Bieberbach dans tous les cas où la géométrie maximale correpondante n'est pas anti de Sitter. Dans le cas des géométries Lorentz-Heisenberg ou Lorentz-SOL, le groupe d'holonomie est, à indice fini près, un réseau cocompact de Heis ou $S O L$, respectivement.

(v) $M$ est géodésiquement complète, sauf si elle est localement modelée sur la géométrie Lorentz-SOL.

Précisons à présent les implications qui existent entre les différents points du théorème précédent. Un résultat essentiel qui permet de passer de la complétude géodésique à la complétude au sens des $(G, G / I)$-structures est le lemme classique suivant usuellement attribué à Thurston [39]:

Lemme 2.2 Soit $M$ une variété qui admet une $(G, G / I)$-structure telle que l'action de $G$ sur $G / I$ préserve une connexion linéaire $\nabla$. Si la connexion induite sur $M$ par $\nabla$ est géodésiquement complète, alors la $(G, G / I)$-structure de M est complète.

Ce résultat, combiné avec le théorème classique de complétude géodésique de HopfRinow [40], implique que les $(G, G / I)$-structures riemanniennes sur des variétés compactes sont automatiquement complètes. En particulier, toute $(G, G)$-structure (où le groupe de Lie $G$ agit sur lui-même par translations) sur une variété compacte $M$ est complète et $M$ s'identifie au quotient de $G$ par un réseau cocompact.

Grâce aux résultats de Carrière et Klingler [7,22] qui affirment que les variétés lorentziennes compactes de courbure sectionnelle constante sont géodésiquement complètes, le lemme 2.2 fournit également la complétude des variétés compactes localement modelées sur les $(G, G / I)$-structures pour lesquelles l'action de $G$ préserve une métrique lorentzienne sur $G / I$ de courbure sectionnelle constante. Le théorème de classification des géométries lorentziennes non-riemanniennes maximales (partie ( $i i)$ du théorème 2.1) permet alors de restreindre l'étude de la complétude aux variétés compactes localement modelées sur les géométries Lorentz-Heisenberg et Lorentz-SOL. La complétude et la rigidité de Bieberbach des réalisations compactes des géométries Lorentz-Heisenberg et Lorentz-SOL se démontre via les propositions 8.1 et 7.1 respectivement. Finalement, on obtient le résultat de complétude de la partie (iii) du théorème principal, qui peut s'énoncer également de la manière suivante:

Corollaire 2.3 Toute variété lorentzienne localement homogène compacte connexe et de dimension 3 est isométrique au quotient (à gauche) d'un espace homogène lorentzien G/I par un sous-groupe discret $\Gamma$ de $G$ agissant proprement et librement.

Mentionnons que, dans le contexte des métriques lorentziennes analytiques de dimension 3, l'homogénéité locale sur un ouvert non vide de $M$ assure l'homogénéité locale sur $M$ [11] et le corollaire précédent s'applique.

Une conséquence du théorème 2.1 est le résultat d'uniformisation suivant: 
Théorème 2.4 Toute variété compacte connexe de dimension 3 qui possède une métrique lorentzienne localement homogène dont le groupe d'isotropie locale est non compact admet un revêtement fini qui possède une métrique lorentzienne de courbure sectionnelle constante (négative ou nulle).

En effet, si la géométrie maximale correspondante n'est pas de courbure sectionnelle constante, celle-ci coïncide alors avec la géométrie Lorentz-Heisenberg, ou bien avec la géométrie Lorentz-SOL. Dans les deux cas, la partie $(i v)$ du théorème 2.1 assure que (à revêtement fini près) la variété $M$ est un quotient (à gauche) de Heis ou de $S O L$ par un réseau. Or, aussi bien $\mathrm{Hei}$, que $S O L$, possèdent des métriques lorentziennes plates invariantes à gauche [35] qui descendent bien sur $M$.

Ainsi, les quotients compacts de la forme $\Gamma \backslash$ Heis portent deux géométries lorentziennes maximales différentes : Minkowski et Lorentz-Heisenberg. Ce phénomène est spécifique à la géométrie non-riemannienne : dans le contexte riemannien il y a unicité de la géométrie maximale portée par les variétés compactes de dimension trois [37].

Par ailleurs, les parties (i) et (iii) du théorème 2.1 permettent de ramener l'étude du problème de complétude géodésique à la complétude géodésique des métriques invariantes à gauche sur les groupes de Lie $\mathbf{R}^{3}, S \widetilde{L(2, \mathbf{R})}$, Heis ou $S O L$ et d'obtenir la partie $(v)$ du théorème 2.1, en utilisant des résultats connus dans ce contexte.

Nous avons vu que la complétude géodésique est acquise dans le cas de courbure sectionnelle constante. La complétude géodésique de la géométrie Lorentz-Heisenberg est démontrée dans la Sect. 4 de [35], où le calcul explicite des géodésiques est présenté. Plus généralement, toutes les métriques lorentziennes invariantes à gauche sur Heisenberg sont géodésiquement complètes [17].

Le manque de complétude géodésique de la métrique Lorentz-SOL est prouvé dans [19].

Finalement le cas des métriques invariantes sur $S \widetilde{L(2, \mathbf{R})}$ est analysé dans [18] (voir aussi [3]) : le résultat des auteurs implique bien qu'on a complétude géodésique dès que l'isotropie n'est pas compacte (voir la preuve du corollaire 5.2).

Par ailleurs, les exemples de [18] et l'exemple de [19] montrent que la complétude géodésique peut tomber en défaut pour certaines métriques lorentziennes invariantes à gauche sur $S \widetilde{L(2, \mathbf{R})}$ ou sur $S O L$ (ici, même en présence d'un groupe d'isotropie non compact). Néanmoins notre résultat de complétude pour les réalisations compactes des $(G, G / I)$ géométries lorentziennes persiste même quand le modèle $G / I$ lui-même n'est pas géodésiquement complet.

Citons également le récent travail [5] qui étudie les tenseurs de courbure des géométries lorentziennes de dimension trois.

En conclusion, notre article classifie les variétés lorentziennes localement homogènes compactes de dimension trois et généralise à ce contexte des résultats connus dans le cadre de courbure sectionnelle constante. Il fournit en même temps un pendant lorentzien à la classification de $\mathrm{W}$. Thurston des géométries riemanniennes de dimension trois.

Organisation de l'article Dans la Sect. 3, nous étudions l'algèbre de Lie des champs de Killing locaux $\mathcal{G}$ d'une variété lorentzienne localement homogène de dimension 3. Comme $\mathcal{G}$ est supposée transitive, la dimension de $\mathcal{G}$ est au moins 3 . Nous montrons que le cas délicat auquel on peut se ramener rapidement est celui où $\mathcal{G}$ est de dimension 4 et l'algèbre d'isotropie locale $\mathcal{I}$ est de dimension 1 (unipotente ou semi-simple). Nous construisons des feuilletages $\mathcal{F}$, totalement géodésiques et dégénérés pour la métrique, grâce à la non-compacité du groupe d'isotropie locale. 
La Sect. 4 présente les géométries lorentziennes invariantes par translations sur les groupes Heis et $S O L$. On y démontre les propositions 1.1 et 1.2.

Dans la Sect. 5, on détermine les géométries dont l'algèbre de Killing contient une copie de $s l(2, \mathbf{R})$. En particulier, on classifie celles qui sont invariantes par translations sur $S \widetilde{L(2, \mathbf{R})}$.

Dans la Sect. 6, on classifie les algèbres résolubles de dimension 4 qui peuvent apparaître comme algèbre de Killing d'une géométrie lorentzienne non-riemannienne. Une étape intermédiaire importante est la détermination de la structure algébrique du stabilisateur d'une feuille de $\mathcal{F}$. On classifie ensuite les modèles algébriques correspondantes $G / I$ sans hypothèse d'existence de réalisations compactes.

Lors de la Sect. 7, on démontre que la seule géométrie lorentzienne non-riemannienne maximale avec $\mathcal{G}$ résoluble de dimension 4 et isotropie contenant un sous-groupe à un paramètre unipotent est Lorentz-SOL. On montre également la complétude et la rigidité de Bieberbach des réalisations compactes de la géométrie Lorentz-SOL.

Dans la Sect. 8, on prouve que dans le cas restant, $\mathcal{G}$ résoluble de dimension 4 et isotropie contenant un sous-groupe à un paramètre semi-simple, la géométrie est Lorentz-Heisenberg. On démontre ici la complétude et la rigidité de Bieberbach des réalisations compactes de la géométrie Lorentz-Heisenberg.

\section{Dynamique lorentzienne locale}

Considérons une métrique lorentzienne $g$ sur une variété $M$. Dans toute la suite de l'article la variété $M$ sera supposée compacte, connexe et de dimension 3 et $g$ sera supposée localement homogène.

Rappelons qu'un champ de vecteurs (local) est dit champ de Killing (local) de $g$ si son flot (local) préserve $g$. La métrique lorentzienne $g$ est dite localement homogène si l'algèbre des champs de Killing locaux $\mathcal{G}$ de $g$ agit transitivement sur $M$. Dans ce cas, la dimension de $\mathcal{G}$ est minorée par la dimension de $M$. La sous-algèbre d'isotropie $\mathcal{I}$, formée par les éléments de $\mathcal{G}$ qui s'annulent en un point $x_{0} \in M$, engendre le pseudo-groupe des isométries locales qui fixent $x_{0}$.

Rappelons qu'une isométrie locale de $g$ est entièrement déterminée par son 1-jet [10,40], ce qui explique que $\mathcal{I}$ s'injecte dans le groupe orthogonal de $\left(T_{x_{0}} M, g_{x_{0}}\right)$. Il en découle que la dimension de $\mathcal{I}$ est inférieure ou égale à la dimension du groupe orthogonal précédent.

Proposition 3.1 À revêtement fini près, toute métrique lorentzienne localement homogène $g$ sur une variété compacte $M$ de dimension 3 est localement modelée sur une unique géométrie maximale $(G, G / I)$, avec $G$ connexe.

i) La dimension de $G$ est $\leq 6$, avec égalité si et seulement si g est de courbure sectionnelle constante.

ii) Si la dimension de $G$ est égale à 3 , alors $M$ est (à revêtement fini près) un quotient $\Gamma \backslash G$, de $G$, par un réseau cocompact $\Gamma$. De plus, l'image réciproque de g sur $G$ est une métrique lorentzienne invariante par translation à gauche.

iii) La dimension de $G$ est différente de 5 (donc I n'est jamais de dimension 2).

Remarque 1 En dimension plus grande, en général, l'existence d'un modèle $G / I$ tombe en défaut, aussi bien dans le contexte riemannien [21,25], que dans le contexte pseudo-riemannien [33].

Démonstration Un modèle local $G / I$ existe si et seulement si le groupe $I$ associé à la sousalgèbre $\mathcal{I}$ de $\mathcal{G}$ est fermé dans l'unique groupe connexe et simplement connexe $G$ associé 
à $\mathcal{G}$ (voir le théorème 1.3 de [33]). Or, d'après un résultat de G. Mostow [30], ceci est vrai dès que la codimension de $\mathcal{I}$ dans $\mathcal{G}$ est $<5$. La codimension de $\mathcal{I}$ dans $\mathcal{G}$ étant ici égale à 3 (la dimension de $M$ ), le théorème de Mostow s'applique. La variété $M$ est alors localement modelée sur une géométrie dont le groupe est le groupe des isométries de $G / I$ (et pas seulement sa composante neutre $G$ ). Comme le groupe des isométries de $G / I$ admet un nombre fini de composantes connexes (en effet, nous verrons au point iii) que le stabilisateur d'un point s'identifie à un sous-groupe algébrique de $O(2,1)$ et admet donc un nombre fini de composantes connexes), un revêtement fini de $M$ est localement modelé sur ( $G, G / I)$.

i) La dimension de $\mathcal{G}$ est bornée supérieurement par la somme de la dimension de $M$ et de la dimension du groupe orthogonal correspondant. En dimension 3, la dimension maximale de $\mathcal{G}$ est donc égale à 6 et elle caractérise les métriques de courbure sectionnelle constante. En effet, dans ce cas $\mathcal{I}$ est de dimension 3 et agit transitivement sur les deux-plans non dégénérés de type temps et sur les deux-plans non dégénérés de type espace contenus dans $T_{x_{0}} M$. Ceci implique, dans un premier temps, que la courbure sectionnelle en $x_{0}$ ne dépend que de $x_{0}$ et l'homogénéité locale permet de conclure que la courbure sectionnelle est constante sur $M$ (pour les notions classiques de géométrie lorentzienne le lecteur pourra consulter [40]).

ii) Dans ce cas $M$ admet une $(G, G)$-structure, avec $G$ agissant par translation à gauche sur lui-même. Le revêtement universel de $M$ s'identifie donc au modèle $G$ et $M$ est isométrique au quotient de $G$ par un réseau cocompact.

iii) La preuve est basée essentiellement sur le fait que le stabilisateur d'une orbite d'une action linéaire algébrique de $\operatorname{PSL}(2, \mathbf{R})$ sur un espace vectoriel de dimension finie est un sous-groupe algébrique qui n'est jamais de dimension 2. Pour la preuve de ce fait il suffit de le constater pour les représentations irréductibles de $\operatorname{PSL}(2, \mathbf{R})$ et de remarquer que, dans le cas général, le stabilisateur d'une orbite est l'intersection des stabilisateurs qui correspondent aux projections de cette orbite sur chaque représentation irréductible.

Considérons l'action de $\mathcal{I}$ sur l'espace tangent $T_{x_{0}} M$ (le groupe d'isotropie locale s'identifie alors à un sous-groupe du groupe orthogonal $O(2,1) \simeq P S L(2, \mathbf{R}))$. Cette action préserve le tenseur courbure de Ricci en $x_{0}$, noté $R i c c i_{x_{0}}$.

Considérons l'action du groupe orthogonal $\operatorname{PSL}(2, \mathbf{R})$ sur l'espace vectoriel $S^{2}\left(T_{x_{0}}^{*} M\right)$ des formes quadratiques sur $T_{x_{0}} M$. L'action préserve la métrique $g_{x_{0}}$ et induit une action de $P S L(2, \mathbf{R})$ sur l'espace vectoriel quotient $S^{2}\left(T_{x_{0}}^{*} M\right) / \mathbf{R} g_{x_{0}}$.

Le groupe d'isotropie locale est contenu dans le stabilisateur de l'élément induit par Ricci $_{x_{0}}$ dans l'espace vectoriel $S^{2}\left(T_{x_{0}}^{*} M\right) / \mathbf{R} g_{x_{0}}$.

Si la dimension du groupe d'isotropie est strictement supérieure à 1 , il s'ensuit que le stabilisateur de la courbure de Ricci est de dimension 3 (on a vu que la dimension ne peut être égale à 2) et que ce stabilisateur coïncide avec tout le groupe orthogonal. Ceci implique que $\operatorname{Ricci}_{x_{0}}=\lambda g_{x_{0}}$ avec $\lambda \in \mathbf{R}$ (la fonction $\lambda$ est constante sur $M$ par homogénéité locale) et notre espace est à courbure sectionnelle constante. Le groupe d'isotropie est alors de dimension 3. On vient de prouver que le groupe d'isotropie n'est jamais de dimension 2 et que donc la dimension de $G$ est $\neq 5$.

Il reste à régler le cas où l'algèbre de Lie $\mathcal{G}$ des champs de Killing est de dimension 4 et l'algèbre d'isotropie $\mathcal{I}$ est de dimension 1 et agit non proprement sur $\mathcal{G} / \mathcal{I}$. Ce sera toute la suite de notre travail qui se concentrera sur cette situation.

La composante neutre du groupe d'isotropie locale $I$ en $x_{0}$ s'identifie avec un sous-groupe à un paramètre du groupe orthogonal $S O(2,1)$. 
Rappelons que l'action de $S O(2,1)$ sur $\left(T_{x_{0}} M, g_{x_{0}}\right)$ est conjuguée à l'action par la représentation adjointe de $\operatorname{PSL}(2, \mathbf{R})$ sur son algèbre de Lie (car cette action préserve la forme de Killing sur $\operatorname{sl}(2, \mathbf{R}))$.

Rappelons également que les sous-groupes à un paramètre de $\operatorname{PSL}(2, \mathbf{R})$ sont conjugués à l'un des sous-groupes suivants:

(1) un sous-groupe elliptique de la forme $\left(\begin{array}{c}\cos t \sin t \\ -\sin t \cos t\end{array}\right)$ qui fixe un vecteur de norme -1 dans $T_{x_{0}} M$;

(2) un sous-groupe unipotent $\left(\begin{array}{ll}1 & t \\ 0 & 1\end{array}\right)$ qui fixe un vecteur isotrope dans $T_{x_{0}} M$;

(3) un sous-groupe semi-simple $\left(\begin{array}{cc}t & 0 \\ 0 & t^{-1}\end{array}\right)$ qui fixe un vecteur de norme 1 dans $T_{x_{0}} M$.

Le cas elliptique sera exclu de notre étude car il représente une géométrie riemannienne. Nous allons nous concentrer donc sur les cas d'isotropie semi-simple ou d'isotropie unipotente.

Base adaptée Dans le cas d'isotropie semi-simple l'action de $\mathcal{I}$ sur $T_{x_{0}} M$ fixe un vecteur $e_{1}$ de norme égale à 1 (uniquement déterminé à revêtement double près). Le plan $e_{1}^{\perp}$ est alors lorentzien et les vecteurs $e_{2}, e_{3} \in e_{1}^{\perp}$ sont définis à constante multiplicative près par les conditions suivantes : $e_{2}, e_{3}$ engendrent les deux directions isotropes du plan $e_{1}^{\perp}$ et $g\left(e_{2}, e_{3}\right)=1$. L'action du temps $t$ du flot de $\mathcal{I}$ s'exprime dans la base $\left(e_{1}, e_{2}, e_{3}\right)$ par $\left(e_{1}, e_{2}, e_{3}\right) \rightarrow\left(e_{1}, e^{t} e_{2}, e^{-t} e_{3}\right)$.

Dans le cas d'isotropie unipotente l'action de $\mathcal{I}$ sur $T_{x_{0}} M$ fixe un vecteur isotrope $e_{1}$ et donc également le plan dégénéré $e_{1}^{\perp}$ (qui contient $e_{1}$ ). Considérons alors des vecteurs $e_{2}$ et $e_{3}$ qui vérifient les relations suivantes : $g\left(e_{1}, e_{2}\right)=0, g\left(e_{2}, e_{2}\right)=1, g\left(e_{3}, e_{3}\right)=0, g\left(e_{2}, e_{3}\right)=0$ et $g\left(e_{3}, e_{1}\right)=1$.

Une telle base sera dite adaptée. Remarquons qu'une base adaptée est entièrement déterminée par le choix du vecteur unitaire $e_{2} \in e_{1}^{\perp}: e_{3}$ sera alors l'unique vecteur isotrope situé dans le plan lorentzien $e_{2}^{\perp}$ et qui est tel que $g\left(e_{3}, e_{1}\right)=1$ (les vecteurs $e_{1}$ et $e_{3}$ engendrent donc les deux directions isotropes de $e_{2}^{\perp}$ ). Le passage d'une base adaptée à une autre se fait par l'action de la différentielle en $x_{0}$ du groupe d'isotropie engendré par $\mathcal{I}$. La matrice de cette différentielle dans la base $\left(e_{1}, e_{2}, e_{3}\right)$ sera alors $\left(\begin{array}{ccc}1 & t & -\frac{t^{2}}{2} \\ 0 & 1 & -t \\ 0 & 0 & 1\end{array}\right)$.

Lemme 3.2 Si $\mathcal{G}$ est de dimension 4, alors (à revêtement fini près) M possède un champ de vecteurs $X$ qui est $\mathcal{G}$-invariant et de divergence nulle.

i) Si I est unipotent, alors $X$ est partout isotrope.

ii) Si I est semi-simple, alors $X$ est un champ de Killing de norme constante égale à 1.

Corollaire 3.3 Si I est semi-simple, $\mathcal{G}$ admet un centre non trivial.

Remarque 2 Dans le cas d'isotropie unipotente le champ de vecteurs fixé par l'isotropie n'est pas toujours de Killing (voir le cas $c \neq 0$ dans la Sect. 7 qui mène à la géométrie Lorentz$\mathrm{SOL})$. Ce phénomène représente une exception lorentzienne car dans le cas des géométries riemanniennes avec un groupe d'isométries de dimension 4, le champ de vecteurs stabilisé par l'isotropie est nécessairement de Killing [39].

Démonstration Il suffit de remarquer que le fibré des repères orthonormées de $M$ admet une réduction au groupe structural $I$ (où l'on identifie $I$ à son image dans $O(2,1)$ par sa représentation d'isotropie). Le groupe $I$ n'est pas nécessairement connexe dans $O(2,1)$, mais il a un 
nombre fini de composantes connexes et, à revêtement fini près de $M$, on peut considérer que $I$ est connexe et conjugué au stabilisateur d'un vecteur de norme constante égale à 1 (cas semi-simple) ou bien de norme constante égale à 0 (cas unipotent). Cette réduction du groupe structural détermine un champ de vecteurs $X$ sur $M$, naturellement invariant par l'action de $\mathcal{G}$, et qui est de norme égale à 1 , si $I$ est semi-simple, ou bien isotrope, si $I$ est unipotent.

Montrons que $X$ est de divergence nulle. Désignons par $\operatorname{div}(X)$ la divergence du champ de vecteurs $X$ par rapport à la forme volume vol induite sur $M$ par la métrique lorentzienne : $L_{X} v o l=\operatorname{div}(X) \cdot v o l$, où $L_{X}$ est la dérivée de Lie dans la direction de $X$. Comme $X$ et vol sont $\mathcal{G}$-invariants, il en découle que la fonction $\operatorname{div}(X)$ est également $\mathcal{G}$-invariante et donc constante égale à $\lambda \in \mathbf{R}$. Notons par $\phi^{t}$ le temps $t$ du flot de $X$ : nous avons alors que $\left(\phi^{t}\right)^{*} v o l=\exp (\lambda t) \cdot v o l$, pour tout $t \in \mathbf{R}$. Comme le flot de $X$ doit préserver $\int_{M} v o l$, il s'ensuit que $\lambda=0$.

ii) Supposons que $I$ est semi-simple et montrons que $X$ est de Killing. On montre d'abord qu'à l'instar de $\mathcal{G}$, l' action de $\phi^{t}$ préserve $X^{\perp}$. En effet, fixons un point $x_{0} \in M$ et considérons son image $\phi^{t}\left(x_{0}\right)$ par le temps $t$ du flot de $X$. Comme $X$ est $\mathcal{G}$-invariant, le flot de $X$ commute avec toutes les isométries locales. En particulier, l'isotropie en $x_{0}$ fixe également les points $\phi^{t}\left(x_{0}\right)$ (autrement dit, deux points qui sont dans la même orbite de $X$ ont la même isotropie locale).

Pour chaque $t$ considérons une isométrie locale $g^{t}$ qui envoie $x_{0}$ sur $\phi^{t}\left(x_{0}\right)$. L'action de $g^{t}$ normalise le sous-groupe à un paramètre d'isotropie en $x_{0}$.

Il en découle que les difféomorphismes locaux $\left(g^{t}\right)^{-1} \circ \phi^{t}$ fixent $x_{0}$ ainsi que le vecteur $X\left(x_{0}\right) \in T_{x_{0}} M$ et normalisent le sous-groupe à un paramètre d'isotropie en $x_{0}$.

Par conséquent, la différentielle $L_{t}$ de $\left(g^{t}\right)^{-1} \circ \phi^{t}$ en $x_{0}$ préserve les sous-espaces vectoriels de $T_{x_{0}} M$ stables par l'action du sous-groupe à un paramètre d'isotropie.

La différentielle $L_{t}$ préserve donc le plan $X\left(x_{0}\right)^{\perp}$ et ses deux droites isotropes. Comme $\operatorname{div}(X)=0$, la différentielle $L_{t}$ préserve le volume. Il en découle que le produit des deux valeurs propres associées aux deux directions isotropes de $X\left(x_{0}\right)^{\perp}$ vaut 1 . Ceci implique que la différentielle de $\left(g^{t}\right)^{-1} \circ \phi^{t}$ en $x_{0}$ est une isométrie et que le flot de $X$ agit par isométries.

Nous avons donc que $X$ est un élément non trivial du centre de $\mathcal{G}$.

Désignons par $\nabla$ la connexion de Levi-Civita de $g$. Nous avons le

Lemme 3.4 Si I est unipotent, alors le champ d'endomorphismes $\nabla . X$ du fibré tangent s'exprime dans une base adaptée sous la forme $\left(\begin{array}{lll}0 & 0 & \alpha \\ 0 & 0 & 0 \\ 0 & 0 & 0\end{array}\right)$, avec $\alpha \in \mathbf{R}$.

De plus, $X$ est de Killing si et seulement si $\alpha=0$.

Démonstration Soit $B$ la matrice de $\nabla . X$ dans une base adaptée de $T_{x_{0}} M$. L'invariance de $\nabla . X$ par l'action de $\mathcal{I}$ implique, en particulier, que la matrice $B$ commute avec $L_{t}=$ $\left(\begin{array}{ccc}1 & t & -\frac{t^{2}}{2} \\ 0 & 1 & -t \\ 0 & 0 & 1\end{array}\right)$, pour tout $t \in \mathbf{R}$. Les espaces propres de chacune des deux matrices sont stables par l'autre. Comme $L_{t}$ ne laisse stable aucune décomposition non triviale de $T_{x_{0}} M$ en somme directe, il s'ensuit que toutes les valeurs propres de $B$ sont égales. Un calcul direct précise la forme de la matrice: $B=\left(\begin{array}{ccc}\lambda & \beta & \alpha \\ 0 & \lambda & -\beta \\ 0 & 0 & \lambda\end{array}\right)$, avec $\alpha, \beta, \lambda \in \mathbf{R}$.

Il suffit de montrer que $\nabla . X$ est nilpotent et que $\beta=0$. 
D'après le lemme 3.2, $\operatorname{div}(X)=0$. Or, la divergence de $X$ en un point $x_{0}$ de $M$ n'est rien d'autre que la trace de l'endomorphisme $(\nabla \cdot X)\left(x_{0}\right)$, égale en occurrence à $3 \lambda$.

Ainsi, $\lambda=0$. Par ailleurs, il sera montré, de manière indépendante, dans la proposition 6.3, que $X$ est parallèle le long de toute courbe tangente à $X^{\perp}$. Ceci implique que $\nabla_{e_{2}} X=0$ et donc que $\beta=0$.

Le champ de vecteurs $X$ est de Killing si et seulement si l'opérateur $\nabla$. $X$ est $g$-antisymétrique [40]. Un opérateur de rang $\leq 1$ étant anti-symétrique si et seulement s'il est identiquement nul, il en découle que $X$ est de Killing si et seulement si $\alpha=0$.

Un phénomène caractéristique à la dynamique lorentzienne, remarqué pour la première fois par M. Gromov dans [16] (voir également l'article de survol [10]) et qui a été amplement utilisé depuis est le fait que la présence d'un groupe d'isotropie (local) non compact implique l'existence de hypersurfaces totalement géodésiques dégénérée pour la métrique.

Le fait remarquable est que dans notre contexte ce feuilletage est régulier :

Lemme 3.5 i) Dans le cas d'isotropie unipotente la variété $M$ possède un feuilletage $\mathcal{F}$ de dimension deux, totalement géodésique et g-dégénéré, dont le plan tangent en chaque point est $X^{\perp}$.

ii) Dans le cas d'isotropie semi-simple la variété $M$ possède deux feuilletages de dimension deux, totalement géodésiques et dégénérées $\mathcal{F}_{1}$ et $\mathcal{F}_{2}$. Le plan tangent à chacun de ces feuilletages est engendré par le champ de vecteurs $X$ et par une des deux droites isotropes de $X^{\perp}$.

L'action de $\mathcal{G}$ préserve chacun de ces feuilletages.

Démonstration L'idée de la démonstration consiste à voir le graphe d'un élément $f$ du groupe d'isotropie locale en $x_{0} \in M$ comme une sous-variété de dimension 3 au voisinage de $\left(x_{0}, x_{0}\right)$ dans $M \times M$. Cette sous-variété est totalement géodésique et isotrope pour la métrique pseudo-riemannienne $g \oplus(-g)$ sur $M \times M$. Si $f_{n}$ est une suite d'éléments du groupe d'isotropie locale qui tend vers l'infini dans $O(2,1)$, alors la suite des graphes de $f_{n}$ converge vers une sous-variété $F^{\prime}$ totalement géodésique et isotrope, mais qui ne représente plus un graphe. Néanmoins l'intersection de $F^{\prime}$ avec l'espace vertical $\left\{x_{0}\right\} \times M$ est de dimension au plus 1, car il s'agit d'une sous-variété isotrope de la variété lorentzienne $M$. Il s'ensuit que la projection $F$ de $F^{\prime}$ sur l'horizontale $M \times\left\{x_{0}\right\}$ s'identifie avec une surface totalement géodésique qui passe par $x_{0}$ et qui est dégénérée. Rappelons ici que le rang et la signature de la restriction de $g$ à une surface totalement géodésique sont invariants par transport parallèle et donc constants.

Dans notre cas, il suffit de considérer une suite d'isométries locales qui se trouve dans le sous-groupe à un paramètre engendré par $\mathcal{I}$. Ces isométries se linéarisent en coordonnées exponentielles et s'expriment dans une base adaptée de $T_{x_{0}} M$ sous la forme présentée précédemment. On constate immédiatement que la limite de nos suites de graphes d'applications linéaires est le plan $X\left(x_{0}\right)^{\perp}$ dans le cas d'isotropie unipotente et les deux plans engendrés par $X\left(x_{0}\right)$ et par chacune des deux directions isotropes de $X\left(x_{0}\right)^{\perp}$ dans le cas d'isotropie semi-simple.

Finissons la preuve dans le cas d'isotropie unipotente.

Nous venons de prouver que par chaque point $x_{0} \in M$ passe une surface totalement géodésique dégénérée tangente à $X\left(x_{0}\right)^{\perp}$. Nous démontrons que cette surface est unique. Supposons par l'absurde qu'il existe une deuxième surface totalement géodésique dégénérée tangente au point $x_{0}$ à un plan dégénéré contenu dans $T_{x_{0}} M$. Comme $\mathcal{I}$ agit transitivement sur les plans dégénérés différents de $X\left(x_{0}\right)^{\perp}$, il en découle que chaque plan dégénéré contenu dans $T_{x_{0}} M$ est tangent à une surface totalement géodésique dégénérée. Ceci implique que la 
métrique lorentzienne $g$ est à courbure sectionnelle constante (voir [42], proposition 3.1) : absurde (car nous sommes dans le cas d'isotropie de dimension 1).

Il s'ensuit que l'unique surface totalement géodésique dégénérée qui passe par $x_{0}$ est en tout point tangente à $X^{\perp}$. Le champ de plans $X^{\perp}$ est intégrable et le feuilletage $\mathcal{F}$ engendré est totalement géodésique et dégénéré.

Comme l'action de $\mathcal{G}$ préserve $X$, elle préserve également $X^{\perp}$ et le feuilletage $\mathcal{F}$. Les mêmes arguments s'appliquent dans le cas d'isotropie semi-simple.

Le stabilisateur $\boldsymbol{H}$ d'une feuille Si $I$ est unipotent, désignons par $\mathcal{H}$ la sous-algèbre de $\mathcal{G}$ qui stabilise la feuille $F=\mathcal{F}\left(x_{0}\right)$ de $x_{0}$ et par $H$ le sous-groupe de $G$ correspondant. Si $I$ est semi-simple, on garde les mêmes notations pour le stabilisateur de $\mathcal{F}_{1}\left(x_{0}\right)$.

Lemme 3.6 Le groupe $H$ est de dimension 3 et agit transitivement sur $\mathcal{F}\left(x_{0}\right)$ (respectivement $\left.\mathcal{F}_{1}\left(x_{0}\right)\right)$. L'isotropie I en $x_{0}$ est contenue dans $H$.

Corollaire 3.7 Les feuilles de $\mathcal{F}\left(\right.$ resp. $\left.\mathcal{F}_{1}\right)$ sont localement modelées sur $(H, H / I)$.

Démonstration Faisons la preuve dans le cas où $I$ est unipotent.

Une isométrie locale de $g$ qui relie deux points $x_{0}$ et $x_{1}$ d'une même feuille $\mathcal{F}\left(x_{0}\right)$ préserve $X$ et également $X^{\perp}$ et envoie donc $\exp _{x_{0}}\left(X^{\perp}\right)$ sur $\exp _{x_{1}}\left(X^{\perp}\right)$. Il en découle que cette isométrie locale stabilise la feuille totalement géodésique $\mathcal{F}\left(x_{0}\right)$ et que, en particulier, l'algèbre d'isotropie $\mathcal{I}$ du point $x_{0}$ est contenue dans l'algèbre $\mathcal{H}$ qui stabilise la feuille $\mathcal{F}\left(x_{0}\right)$. Comme $\mathcal{G}$ agit transitivement sur $M$, la remarque précédente implique que $\mathcal{H}$ agit transitivement sur $\mathcal{F}\left(x_{0}\right)$ (avec une isotropie de dimension 1) ce qui implique que $\mathcal{H}$ est de dimension 3.

Remarquons que la restriction de $\mathcal{H}$ à une feuille $F$ de $\mathcal{F}$ est un isomorphisme d'algèbres de Lie. Ceci est dû au fait qu'une isométrie locale de $g$ est complètement déterminée par sa restriction à un sous-ensemble invariant totalement géodésique de dimension 2 (il suffit de linéariser l'isométrie en coordonnées exponentielles et de vérifier ce fait pour les applications linéaires).

Dans la suite de l'article nous allons également désigner par $X$ et par $\mathcal{F}$, les objets correspondants sur le modèle $G / I$.

\section{Géométrie des métriques invariantes à gauche}

Dans cette section, nous examinons la géométrie de certaines métriques lorentziennes invariantes par translations à gauche sur des groupes de Lie unimodulaires de dimension 3. Cette étude a été initiée par J. Milnor [27] dans le cas riemannien et poursuivie dans le cadre lorentzien $[8,32,34]$. Comme les métriques lorentziennes qui nous intéressent sont précisément celles de courbure sectionnelle non constante et dont le groupe d'isotropie locale est non compact, les groupes $\mathbf{R}^{3}, S^{3}$ et le groupe des déplacements du plan euclidien seront exclus de notre étude. Il reste à examiner les groupes $H e i s, S O L$ et $S \widetilde{L(2, \mathbf{R})}$. Le cas $S \widetilde{L(2, \mathbf{R})}$ fera l'objet de la proposition 5.1, en Sect. 5.

\subsection{Cas du groupe Heis}

Nous décrivons ici les métriques lorentziennes invariantes à gauche sur Heisenberg et démontrons au passage la proposition 1.1. 
Démonstration L'algèbre de Lie heis est engendrée par un élément central $X^{\prime}$ et par deux éléments $Z$ et $T$ tels que $[Z, T]=X^{\prime}$. Les automorphismes de l'algèbre de Lie préservent le centre et envoient donc l'élément $X^{\prime}$ sur un multiple de la forme $\lambda X^{\prime}$, avec $\lambda \in \mathbf{R}^{*}$. Un tel automorphisme agit sur le plan heis $/ \mathbf{R} X^{\prime} \simeq \mathbf{R}^{2}$ par un automorphisme de déterminant égal à $\lambda$. Inversement, toutes ces transformations sont bien des automorphismes de l'algèbre de Lie heis.

Il est montré dans [34] et [35] que, modulo automorphisme de heis, il existe trois classes de métriques lorentziennes invariantes sur Heis selon que l'élément $X^{\prime}$ est de norme strictement négative, nulle ou bien strictement positive. Pour les résultats suivants, qui décrivent ces métriques, nous renvoyons le lecteur à [34] et [35].

- Dans le cas où $X^{\prime}$ est de norme nulle, la métrique est plate.

- Quand $X^{\prime}$ est de norme constante égale à -1, son orthogonal est le plan riemannien engendré par $Z$ et $T$ (à automorphisme près) et le sous-groupe à un paramètre d'automorphismes de heis qui stabilise $X^{\prime}$ et agit par rotations euclidiennes sur le plan engendré par $Z$ et $T$ constitue un sous-groupe à un paramètre (elliptique) d'isométries. Le groupe des isométries est dans ce cas de dimension au moins 4 et sa composante connexe de l'identité contient le groupe engendrée par les translations à gauche et par le sous-groupe à un paramètre d'automorphismes de Heis qui intègre le sous-groupe à un paramètre d'isotropie précédent (agissant par automorphismes de heis). Par ailleurs, le théorème 4.1 de [34] montre que le groupe des isométries est de dimension égale à 4 et, par conséquent, sa composante neutre coïncide avec le groupe précédent dont l'isotropie est un sous-groupe à un paramètre elliptique. Il s'ensuit que cet exemple est riemannien.

- Le cas intéressant à notre sens et qui fournit la géométrie Lorentz-Heisenberg est celui où $X^{\prime}$ est de norme constante positive. Dans ce cas, quitte à appliquer un automorphisme de heis on peut supposer que $X^{\prime}$ est le plan lorentzien engendré par les droites isotropes portées par $Z$ et $T$. En appliquant finalement un automorphisme de la forme $\left(X^{\prime}, Z, T\right) \rightarrow\left(\lambda^{2} X^{\prime}, \lambda Z, \lambda T\right)$, avec $\lambda \in \mathbf{R}^{*}$, on peut rendre $g$ multiple de l'unique métrique qui attribue à $X^{\prime}$ la norme 1 et qui est telle que $g(Z, T)=1$.

Le groupe des isométries de cette famille de métriques est le même. Il est de dimension 4, il contient Heis et l'isotropie contient le sous-groupe à un paramètre d'automorphismes de heis qui fixe $X^{\prime}$ et qui agit sur le plan lorentzien engendré par $Z$ et $T$ par les matrices $\left(\begin{array}{cc}e^{t} & 0 \\ 0 & e^{-t}\end{array}\right)$. Le groupe d'isotropie contient alors un sous-groupe à un paramètre semi-simple et on est en présence d'une géométrie lorentzienne non-riemannienne dont la composante neutre du groupe des isométries est $G=\mathbf{R} \ltimes H e i s$, où l'action du facteur $\mathbf{R}$ qui engendre l'isotropie sur Heis vient d'être explicitée.

Montrons que cette géométrie est maximale.

D'après le théorème 2 de [35], la seule métrique lorentzienne invariante à gauche sur Heis qui admet un groupe d'isométries de dimension strictement plus grande que 4 est la métrique plate qui attribue à l'élément central $X^{\prime}$ la norme nulle. Par ailleurs, les seules formes quadratiques sur heis qui sont invariantes par le sous-groupe à un paramètre $\left(X^{\prime}, Z, T\right) \rightarrow\left(X^{\prime}, e^{t} Z, e^{-t} T\right)$ sont de la forme $g=\alpha d X^{\prime 2}+\beta d Z d T$, avec $\alpha, \beta \in \mathbf{R}$. Si, de plus, $g$ est non dégénérée, alors $\alpha \neq 0$ et $X^{\prime}$ n'est pas isotrope (la métrique lorentzienne correspondante sur Heis n'est jamais plate). Par conséquent, le groupe $G=\mathbf{R} \ltimes$ Heis n'est pas contenu dans le groupe des isométries de la métrique plate et notre géométrie est maximale. 


\subsection{Cas du groupe $S O L$}

Rappelons que l'algèbre de Lie sol est engendrée par $\left\{X^{\prime}, Z, T\right\}$, avec les éléments $X^{\prime}$ et $Z$ qui commutent et les deux crochets non-nuls $\left[T, X^{\prime}\right]=X^{\prime}$ et $[T, Z]=-Z$.

Nous démontrons à présent la proposition 1.2.

Démonstration Les automorphismes de $s o l$ agissent sur l'algèbre dérivée $\mathbf{R}^{2}=\mathbf{R} X^{\prime} \oplus$ $\mathbf{R} Z$ en préservant la décomposition en espaces propres de l'opérateur $a d(T)$. Cette action se fait donc par homothétie sur chacune des droites $\mathbf{R} X^{\prime}$ et $\mathbf{R} Z$. Par ailleurs, un automorphisme de $s o l$ envoie $T$ sur la somme de $T$ avec un élément de l'algèbre dérivée. Inversement tout isomorphisme de l'espace vectoriel sol de la forme précédente est un automorphisme de $\mathrm{sol}$.

Considérons une métrique lorentzienne $g$, invariante à gauche sur $S O L$, qui rend l'algèbre dérivée dégénérée et l'une des deux directions propres de $\operatorname{ad}(T)$ isotrope.

Pour fixer les idées, supposons que $X^{\prime}$ engendre l'unique direction isotrope de l'algèbre dérivée et donc que $\mathbf{R} X^{\prime} \oplus \mathbf{R} Z$ coïncide avec $X^{\prime}$. Quitte à appliquer une homothétie sur la direction $\mathbf{R} Z$, on peut supposer que $Z$ est de norme égale à 1 . En additionnant à $T$ un multiple de $Z$, on peut considérer que $T$ est orthogonal à $Z$. On applique ensuite une homothétie sur la droite engendrée par $X^{\prime}$ de manière à avoir $g\left(T, X^{\prime}\right)=1$ (ceci est possible car le plan $Z^{\perp}$, engendré par $X^{\prime}$ et $T$, est lorentzien et donc $g\left(T, X^{\prime}\right)$ est non nul). Finalement, on ajoute à $T$ un multiple de $X^{\prime}$, de manière à rendre $T$ de norme égale à 0 (tout en préservant l'orthogonalité entre $T$ et $Z$ ). Nous venons de prouver l'unicité de $g$ (à automorphisme près).

Nous montrons à présent que cette métrique n'est pas plate. Un calcul direct implique que $\nabla_{Z} T=Z, \nabla_{T} Z=0, \nabla_{T} T=-T, \nabla_{X^{\prime}} X^{\prime}=0, \nabla_{Z} Z=-X^{\prime}, \nabla_{X^{\prime}} Z=\nabla_{Z} X^{\prime}=$ $0, \nabla_{X^{\prime}} T=0$ et $\nabla_{T} X^{\prime}=X^{\prime}$.

Ceci donne que $R(T, Z)$ est un endomorphisme non nul qui s'exprime dans la base $\left(X^{\prime}, Z, T\right)$ par la matrice $\left(\begin{array}{ccc}0 & -2 & 0 \\ 0 & 0 & 2 \\ 0 & 0 & 0\end{array}\right)$. La métrique n'est donc pas plate.

Maintenant nous prouvons que le groupe des isométries de la géométrie Lorentz-SOL est de dimension 4. Pour cela nous construisons la métrique précédente d'une manière différente. Considérons l'algèbre de Lie heis engendrée par trois éléments $Y, X^{\prime}, Z$, avec $X^{\prime}$ élément central et $[Y, Z]=X^{\prime}$. Considérons l'action d'un quatrième élément $T$ sur heis donnée, dans la base $\left(X^{\prime}, Z, Y\right)$, par la matrice $\operatorname{ad}(T)=\left(\begin{array}{ccc}1 & 0 & 0 \\ 0 & -1 & 1 \\ 0 & 0 & 2\end{array}\right)$.

Cette action est bien une dérivation, ce qui implique que les éléments $\left\{T, X^{\prime}, Z, Y\right\}$ engendrent une algèbre de Lie $\mathcal{G}$ isomorphe à l'algèbre de Lie du produit semi-direct $G=$ $\mathbf{R} \ltimes$ Heis, où l'action du facteur $\mathbf{R}$, engendré par $T$, sur Heis vient d'être explicitée. Le sous-groupe à un paramètre $I$ engendré par $Y$ est fermé dans $G$ (voir la proposition 3.1). L'espace homogène $G / I$ est lorentzien car l'action de $\operatorname{ad}(Y)$ sur $\mathcal{G} / \mathcal{I}$ s'exprime, dans la base $\left(X^{\prime}, Z, T\right)$, par la matrice $\operatorname{ad}(Y)=\left(\begin{array}{ccc}0 & 1 & 0 \\ 0 & 0 & -1 \\ 0 & 0 & 0\end{array}\right)$, qui engendre un sous-groupe à un paramètre unipotent préservant une métrique lorentzienne pour laquelle $X^{\prime}$ est isotrope et le plan engendré par $X^{\prime}$ et $Z$ dégénéré. Par ailleurs, l'isotropie $I$ est engendrée par $\mathbf{R} Y$ qui intersecte trivialement l'algèbre de Lie sol engendrée par $\left\{T, X^{\prime}, Z\right\}$. Il en découle que l'action du groupe $S O L$ sur $G / I$ est libre et transitive et la métrique précédente s'identifie à l'unique métrique invariante à gauche sur $S O L$ qui rend $X^{\prime}$ isotrope et l'algèbre dérivée $\mathbf{R} X^{\prime} \oplus \mathbf{R} Z$ dégénérée. On vient de démontrer que cette métrique admet une isotropie non 
triviale unipotente et que son groupe des isométries est de dimension au moins 4. La géométrie Lorentz-SOL est donc non-riemannienne. Comme la métrique n'est pas de courbure sectionnelle constante, la proposition 3.1 implique que le groupe des isométries est de dimension égale à 4 et sa composante neutre coïncide avec $G=\mathbf{R} \ltimes H e i s$. Mentionnons que, contrairement à la géométrie Lorentz-Heisenberg, ici le facteur Heis contient l'isotropie $\mathbf{R} Y$ et n'agit donc pas librement.

Le groupe des isométries de la géométrie Lorentz-SOL n'est contenu strictement dans le groupe des isométries d'aucune autre métrique invariante sur $S O L$. En effet, le groupe d'isotropie $I$ agit de manière unipotente sur le plan engendré par $X^{\prime}$ et $Z$. Ceci implique que toute métrique lorentzienne invariante à gauche sur $S O L$, qui est préservée par $I$, rend l'algèbre dérivée dégénérée et la direction $\mathbf{R} X^{\prime}$ isotrope. Or, on a vu que ces métriques coïncident nécessairement (à automorphisme près) avec la métrique Lorentz-SOL. La géométrie Lorentz-SOL est donc maximale.

Le groupe $S O L$ admet également des métriques plates invariantes par translations. Pour s'en convaincre, il suffit d'exhiber une copie de $S O L$ dans le groupe des isométries de Minkowski, qui agit simplement transitivement. Nous ne construirons pas un tel exemple ici, car une métrique plate explicite sur $S O L$ apparaîtra naturellement en Sect. 8, au cours de la preuve de la proposition 8.2.

\section{Modèles algébriques $S L(2, \mathrm{R})$-invariants}

Nous classifions ici les géométries lorentziennes non-riemanniennes de la forme $G / I$, avec $G$ groupe de Lie de dimension 4 non résoluble et isotropie $I$ de dimension 1 .

Proposition 5.1 Si $\mathcal{G}$ a une partie semi-simple non triviale, alors $\mathcal{G}$ est l'algèbre de Lie de $\mathbf{R} \times S \widetilde{L(2, \mathbf{R})}$ et $g$ est isométrique à :

(1) une métrique lorentzienne sur $\widehat{S(2, \mathbf{R})}$ invariante par les translations à gauche et par un sous-groupe à un paramètre unipotent (respectivement semi-simple) de translations à droite. L'isotropie I est la diagonale du produit $\mathbf{R} \times S \widetilde{L(2, \mathbf{R})}$. La géométrie maximale correspondante est celle de courbure sectionnelle constante négative donnée par la forme de Killing.

(2) la géométrie produit de $\mathbf{R}$ et du plan de Sitter de dimension 2, i.e. la surface lorentzienne homogène simplement connexe de courbure sectionnelle constante non nulle. L'isotropie I est incluse dans le facteur $\widehat{S L(2, \mathbf{R})}$ et est conjuguée à un sous-groupe à un paramètre semi-simple.

Remarque 3 On montrera à la proposition 8.4 (Sect. 8) que la géométrie produit du cas (2) ne se réalise pas sur des variétés compactes.

Corollaire 5.2 Toute réalisation compacte d'une géométrie du type précédent admet un revêtement universel isométrique à $S \widetilde{L(2, \mathbf{R})}$ muni d'une métrique invariante à gauche et par un sous-groupe à un paramètre (semi-simple ou unipotent) de translations à droite. Ces métriques sont géodésiquement complètes.

Passons à la preuve de la proposition.

Démonstration Comme $\mathcal{G}$ est de dimension 4 et qu'il n'existe pas de groupe de Lie semisimple de dimension 4 , il s'ensuit que $\mathcal{G}=\mathbf{R} \oplus \mathcal{G}_{1}$, où $\mathcal{G}_{1}$ est une algèbre de Lie semi-simple 
de dimension 3 [20]. L'algèbre de Lie $\mathcal{G}_{1}$ est alors isomorphe à $s l(2, \mathbf{R})$ ou à l'algèbre de Lie de $S^{3}$, ù $S^{3}$ est la sphère de dimension 3 avec sa structure canonique de groupe de Lie.

(1) Considérons d'abord le cas où $\mathcal{G}_{1}$ agit librement transitivement sur $G / I$ et donc $g$ s'identifie avec une métrique invariante par translations sur le groupe de Lie associé $G_{1}$.

Si $G_{1}$ est $S^{3}$, le modèle $G / I$ s'identifie à $S^{3}$ muni d'une métrique lorentzienne invariante par translation. Comme $S^{3}$ est simplement connexe, d'après un résultat de [9] le groupe des isométries $G$ est compact. Ceci implique que $I$ est compact et qu'il s'agit donc d'une géométrie riemannienne.

Il reste à régler le cas où $G_{1}$ est $\widehat{S L(2, \mathbf{R})}$. Considérons $\left(X^{\prime}, Y, Z\right)$ une base de l'algèbre de Lie $\operatorname{sl}(2, \mathbf{R})$ avec les crochets de Lie : $\left[X^{\prime}, Y\right]=Y,\left[X^{\prime}, Z\right]=-Z$ et $[Z, Y]=2 X^{\prime}$.

Complétons cette base en une base de $\mathcal{G}$ en ajoutant un générateur $T$ de $\mathcal{I}$.

Considérons l'action du groupe d'isotropie $I$ en un point $x_{0}$ sur l'idéal $\operatorname{sl}(2, \mathbf{R})$ qui s'identifie à $T_{x_{0}} M$. Cette action se fait par isomorphisme de l'algèbre de Lie $\operatorname{sl}(2, \mathbf{R})$ et doit fixer un vecteur de $\operatorname{sl}(2, \mathbf{R})$. Quitte à opérer un changement de base par un automorphisme intérieur de $\operatorname{sl}(2, \mathbf{R})$ on peut supposer que le vecteur stabilisé par $I$ coïncide avec $X^{\prime}$ (s'il est de norme strictement positive pour la forme de Killing) ou bien avec $Y$ (s'il est de norme nulle pour la forme de Killing). Rappelons que le cas où le champ de vecteurs stabilisé par $I$ est de norme strictement négative correspond à une géométrie de type non-riemannien.

Les seuls isomorphismes de l'algèbre de Lie $\operatorname{sl}(2, \mathbf{R})$ qui fixent $X^{\prime}$ étant de la forme $\left(X^{\prime}, Y, Z\right) \rightarrow\left(X^{\prime}, e^{t} Y, e^{-t} Z\right.$ ), il en découle que l'action de $I$ (donc de $T$ ) coïncide avec celle de $\operatorname{ad}\left(X^{\prime}\right)$. Le quatrième champ de Killing $T$ est alors le sous-groupe à un paramètre de $S \widetilde{L(2, \mathbf{R})}$ engendré par $X^{\prime}$ et plongé diagonalement dans $S \widetilde{L(2, \mathbf{R})} \times \widehat{S L(2, \mathbf{R})}$.

Nous venons de démontrer que $G=\mathbf{R} \times S \widetilde{L(2, \mathbf{R})}$ : la composante connexe de l'identité du groupe des isométries est engendrée par toutes les translations à gauche et par le sous-groupe à un paramètre des translations à droite engendré par $X^{\prime}$.

De la même manière on constate que tous les isomorphismes de l'algèbres de Lie $s l(2, \mathbf{R})$ qui fixent $Y$ sont engendrés par l'action adjointe de $Y$. Dans ce cas la composante connexe de l'identité du groupe des isométries est engendrée par toutes les translations à gauche et par le sous-groupe à un paramètre des translations à droite engendré par $Y$.

Dans les deux cas $G$ est un sous-groupe de $S \widetilde{L(2, \mathbf{R})} \times \widetilde{S(2, \mathbf{R})}$ qui est la composante connexe de l'identité du groupe des isométries de la métrique lorentzienne à courbure sectionnelle constante négative obtenue en considérant la métrique de Kill-

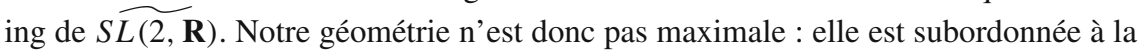
géométrie de courbure sectionnelle constante négative.

(2) Supposons que $\mathcal{I}$ est contenu dans $\mathcal{G}_{1}$.

Ceci implique que les orbites de l'action de $\mathcal{G}_{1}$ sont de dimension 2 et que l'action de l'isotropie préserve une decomposition non triviale de l'espace tangent à $M$ (induite par la décomposition, $a d(I)$-invariante, $\mathcal{G}=\mathbf{R} \oplus \mathcal{G}_{1}$ ). Il s'ensuit que l'isotropie $I$ est semi-simple, que le champ $X$ est engendré par le facteur $\mathbf{R}$ et que l'espace tangent aux orbites de $\mathcal{G}_{1}$ est $X^{\perp}$.

Les orbites de $\mathcal{G}_{1}$ sont alors des surfaces lorentziennes homogènes et donc de courbure sectionnelle constante. Or, l'algèbre de Lie de $S^{3}$ n'est pas l'algèbre de Killing d'une telle surface, tandis que $\operatorname{sl}(2, \mathbf{R})$ se réalise bien comme l'algèbre de Killing du plan de Sitter. 
Nous sommes alors dans le cas de la géométrie produit décrite au point (2), où l'isotropie est conjuguée dans $S \widetilde{L(2, \mathbf{R})}$ à un sous-groupe à un paramètre semi-simple.

Nous passons à la preuve du corollaire 5.2.

Démonstration Par la proposition 5.1, l'action de $G$ sur le modèle $G / I$ préserve une métrique de courbure sectionnelle constante négative. Le lemme 2.2 et le résultat de [22] impliquent que la ( $G, G / I)$-structure de $M$ est complète.

Pour montrer que $M$ est géodésiquement complète, il suffit alors de montrer que le modèle $G / I$ est géodésiquement complet. On utilisera les résultats de [18] sur la complétude géodésique des métriques lorentziennes invariantes à gauche sur $S \widetilde{L(2, \mathbf{R})}$.

Exprimons la forme quadratique $g$ par rapport à la forme de Killing $q$ de $\operatorname{sl}(2, \mathbf{R})$ : $g(u, v)=q(\phi(u), v)$, où $\phi$ est un endomorphisme $q$-symétrique de $\operatorname{sl}(2, \mathbf{R})$ et $u, v \in$ $s l(2, \mathbf{R})$. De plus, $\phi$ est invariant par l'action d'un sous-groupe à un paramètre semi-simple ou unipotent.

Si le sous-groupe à un paramètre est semi-simple, il s'ensuit que $\phi$ laisse stables les espaces propres du sous-groupe à un paramètre semi-simple et il est donc diagonalisable. On vérifie que $\phi$ est $q$-symétrique si et seulement si les deux valeurs propres qui correspondent aux deux vecteurs propres $q$-isotropes sont égales. Il en découle que $\phi$ admet un espace propre de dimension au moins 2 et on se trouve dans le cas (a) d'application du théorème 4 de [18] (voir aussi [3]), qui assure la complétude géodésique de $g$.

Dans le cas restant, le groupe à un paramètre qui laisse invariant $g$ (et donc également $\phi$ ) est unipotent et $\phi$ s'exprime dans une base adaptée (voir la preuve du lemme 3.4) par une matrice de la forme $\left(\begin{array}{ccc}\lambda & \beta & \alpha \\ 0 & \lambda & -\beta \\ 0 & 0 & \lambda\end{array}\right)$, avec $\alpha, \beta, \lambda \in \mathbf{R}$.

Cet endomorphisme est $q$-symétrique si et seulement si $\beta=0$. Il s'ensuit que $\phi$ admet un espace propre de dimension au moins deux et on conclut comme avant.

\section{Modèles algébriques avec $G$ résoluble}

Dans cette section on classifie les géométries lorentziennes non-riemanniennes $G / I$, avec $G$ résoluble de dimension 4 et $I$ de dimension 1.

Nous déterminons d'abord le sous-groupe $H$ (stabilisateur d'une feuille):

\section{Proposition $6.1 \quad$ i) L'algèbre dérivée $[\mathcal{H}, \mathcal{H}]$ est isomorphe à $\mathbf{R}$.}

ii) Le groupe $H$ est isomorphe au groupe de Heisenberg ou bien au produit $H=\mathbf{R} \times A G$, où $A G$ est le groupe affine.

Avant de passer à la preuve rappelons que le groupe affine de la droite $A G$ est un groupe de Lie de dimension 2 qui peut être vu comme l'ensemble des couples $(a, b) \in \mathbf{R}^{2}$ muni de la multiplication $(a, b) \cdot\left(a^{\prime}, b^{\prime}\right)=\left(a+a^{\prime}, \exp (a) b^{\prime}+b\right)$.

Démonstration i) Rappelons que l'algèbre dérivée d'une algèbre résoluble est toujours nilpotente. Or, $\mathcal{H}$ est supposée résoluble et donc $[\mathcal{H}, \mathcal{H}]$ est une algèbre nilpotente de dimension strictement inférieure à 3 .

L'algèbre dérivée $[\mathcal{H}, \mathcal{H}]$ ne peut être triviale. En effet, sinon $\mathcal{H}$ est abélienne et donc l'action de l'isotropie $\mathcal{I} \subset \mathcal{H}$ sur $T_{x_{0}} F \simeq \mathcal{H} / \mathcal{I}$ est triviale. Ceci est absurde car l'on a vu qu'un élément de $\mathcal{G}$ agissant trivialement sur $F$ est trivial. 
Il en découle que $[\mathcal{H}, \mathcal{H}]$ est isomorphe à $\mathbf{R}$ ou à $\mathbf{R}^{2}$. Supposons par l'absurde que l'algèbre dérivée est isomorphe à $\mathbf{R}^{2}$.

On démontre d'abord que $\mathcal{I}$ est inclus dans $[\mathcal{H}, \mathcal{H}]$. Supposons par l'absurde le contraire. Alors $\mathbf{R}^{2}$ agit par isométries sur chaque feuille $F$ du feuilletage $\mathcal{F}$. Nous démontrons alors que la restriction de la connexion $\nabla$ à $F$ est plate. Dans les coordonnées locales $(x, h)$ de $F$ données par l'action de $\mathbf{R}^{2}$, l'expression de $g$ est $d h^{2}$. Dans ces coordonnées, $X$ est également invariant par translations et est de la forme $\frac{\partial}{\partial x}$, si $I$ est unipotent et de la forme $\frac{\partial}{\partial h}$, si $I$ est semi-simple.

Un calcul simple montre que toute connexion sans torsion $\nabla$, invariante par translations sur $\mathbf{R}^{2}$, et compatible avec $d h^{2}$ est donnée par $\nabla_{\frac{\partial}{\partial h}} \frac{\partial}{\partial h}=a \frac{\partial}{\partial x}, \nabla_{\frac{\partial}{\partial x}} \frac{\partial}{\partial x}=b \frac{\partial}{\partial x}$ and $\nabla_{\frac{\partial}{\partial h}} \frac{\partial}{\partial x}=\nabla_{\frac{\partial}{\partial x}} \frac{\partial}{\partial h}=c \frac{\partial}{\partial x}$, avec $a, b, c \in \mathbf{R}$. L'invariance de $\nabla$ par $I$ implique qu'au moins deux des paramètres $a, b$ et $c$ s'annulent. Ceci implique que la courbure de $\nabla$ s'annule.

Par conséquent, le groupe des isométries de ce modèle est contenu dans le groupe affine de $\mathbf{R}^{2}$. Il coïncide avec $\mathbf{R} \ltimes \mathbf{R}^{2}$, où l'action de l'isotropie $I \simeq \mathbf{R}$ sur $\mathbf{R}^{2}$ est donnée par $\left(\begin{array}{ll}1 & t \\ 0 & 1\end{array}\right)$, si $I$ est unipotent, ou par $\left(\begin{array}{ll}e^{t} & 0 \\ 0 & 1\end{array}\right)$, si $I$ est semi-simple. Il s'ensuit que $H$ est isomorphe, ou bien à Heisenberg, ou bien à $A G \times \mathbf{R}$. Dans les deux cas son groupe dérivé est de dimension un : absurde.

Il en découle donc que l'algèbre d'isotropie $\mathcal{I}$ est incluse dans l'algèbre dérivée $[\mathcal{H}, \mathcal{H}]$. Les orbites de $[\mathcal{H}, \mathcal{H}]$ sur $F$ seront alors de dimension un.

Nous démontrons que ces orbites sont les courbes intégrales sur $F$ de l'unique direction isotrope et que l'isotropie $I$ est unipotente. Pour cela, désignons par $Y$ un générateur de l'algèbre d'isotropie, par $\left(Y, X^{\prime}\right)$ une base de l'algèbre commutative $[\mathcal{H}, \mathcal{H}]$ et finalement $\operatorname{par}\left(Y, X^{\prime}, Z\right)$ une base de $\mathcal{H}$. Comme $\operatorname{ad}(Y) \cdot \mathcal{H} \subset[\mathcal{H}, \mathcal{H}]$ et que $[\mathcal{H}, \mathcal{H}]$ est abélienne, il s'ensuit que $a d(Y) \cdot X^{\prime}=0$ et $a d(Y) \cdot Z=a X^{\prime}(\bmod Y)$. Donc, l'action de $\operatorname{ad}(Y)$ sur l'espace tangent à $F$ est unipotente et admet $X^{\prime}$ comme unique direction (isotrope) propre. Or, $X^{\prime}$ engendre bien l'espace tangent aux orbites de $[\mathcal{H}, \mathcal{H}]$ sur $F$.

Désignons par $\mathcal{L}$ l'algèbre dérivée de $\mathcal{G}$. Nous avons les inclusions $\mathcal{I} \subset[\mathcal{H}, \mathcal{H}] \subset \mathcal{L}$. La dimension de $\mathcal{L}$ est 2 ou 3 et les orbites de l'action de $\mathcal{L}$ sur le modèle $G / I$ sont de dimension respectivement 1 ou 2 .

Traitons d'abord le cas où $\mathcal{L}$ est de dimension 3 et ses orbites sont donc de dimension 2. Comme l'unique 2-plan de $T_{x_{0}} G / I$ préservé par l'isotropie unipotente est $X^{\prime \perp}$, il en découle que les orbites de $\mathcal{H}$ et $\mathcal{L}$ coïncident. Ceci est absurde car $\mathcal{L}$ est nilpotente (en tant qu'algèbre dérivée d'une algèbre résoluble) et pas $\mathcal{H}$ (son algèbre dérivée étant de dimension 2).

Il reste à traiter le cas où $\mathcal{L}$ est de dimension 2. Dans ce cas $\mathcal{L}=[\mathcal{H}, \mathcal{H}]$. L'image de $\mathcal{G}$ par l'action de l'isotropie $\operatorname{ad}(Y)$ est alors incluse dans $\mathcal{L}$. Cette image est donc de dimension au plus 2 et, par conséquent, de dimension au plus 1 dans $\mathcal{G} / \mathcal{I}$ qui s'identifie à $T_{x_{0}} G / I$. Ceci est absurde car $a d(Y)$ doit être de rang 2 (comme on l'a vu à la Sect. 3 en considérant des bases adaptées). On vient de montrer que $[\mathcal{H}, \mathcal{H}]$ est de dimension 1 .

ii) Considérons un générateur $Z$ de l'algèbre $[\mathcal{H}, \mathcal{H}]$ et son application adjointe $\operatorname{ad}(Z)$ : $\mathcal{H} \rightarrow \mathbf{R Z}$. Si l'application précédente est identiquement nulle, autrement dit si l'élément $Z$ est central dans $\mathcal{H}$, alors $\mathcal{H}$ est nilpotente et $H$ est isomorphe au groupe de Heisenberg. 
Sinon, soit $X^{\prime}$ un générateur du noyau de $a d(Z)$ et $Y$ un élément de $\mathcal{H}$ tel que $Y, X^{\prime}, Z$ engendrent $\mathcal{H}$. Comme $Z$ n'est pas central, on peut supposer que $[Y, Z]=$ $Z$. Nous avons que $\left[X^{\prime}, Y\right]=\alpha Z$, pour $\alpha \in \mathbf{R}$.

Si $\alpha=0, H=\mathbf{R} \times A G$, le centre étant engendré par $X^{\prime}$ et le deuxième facteur par $\mathbf{R} Z \oplus \mathbf{R} Y$.

Si $\alpha \neq 0$, il suffit de changer $X^{\prime}$ en $X^{\prime}+\alpha Z$ pour se ramener au cas précédent.

6.1 Cas : $H=\mathbf{R} \times A G$

Proposition 6.2 Si $H=\mathbf{R} \times A G$, l'isotropie I est semi-simple et engendrée par le générateur des homothéties dans AG. Le groupe G est l'un des trois groupes suivants :

(1) $G=\mathbf{R} \times S O L$,

(2) $G=\mathbf{R} \ltimes$ Heis,

(3) $G=\mathbf{R}^{2} \ltimes \mathbf{R}^{2}$.

Dans le cas (2) l'action du premier facteur sur Heis, dans une base $\left(X^{\prime}, Z, T\right)$ de heis formée par l'élément central $X^{\prime}$ et telle que $[T, Z]=X^{\prime}$, s'exprime par $\left(X^{\prime}, Z, T\right) \rightarrow$ $\left(X^{\prime}, e^{t} Z, e^{-t} T\right)$.

Dans le cas (3) l'action de la première copie de $\mathbf{R}^{2}$ sur la deuxième copie de $\mathbf{R}^{2}$ est donnée par les matrices $\left(\begin{array}{cc}e^{t} & 0 \\ 0 & e^{-t}\end{array}\right)$ et $\left(\begin{array}{cc}1 & 0 \\ 0 & e^{-t}\end{array}\right)$.

Remarque 4 Comme le centre de $\mathcal{G}=\mathbf{R}^{2} \ltimes \mathbf{R}^{2}$ est trivial, le lemme 3.2 impliquera que cette géométrie ne se réalise pas sur des variétés compactes.

Démonstration Pour fixer les idées supposons que $X^{\prime}$ est un élément central non trivial de $\mathcal{H}$ et que $Y$ et $Z$ engendrent l'algèbre de Lie du groupe affine : $[Y, Z]=Z$. Alors $X^{\prime}, Y, Z$ engendrent $\mathcal{H}$ et désignons par $T$ un quatrième générateur de $\mathcal{G}$.

Nous montrons que, quitte à appliquer un automorphisme de $\mathcal{H}$ qui envoie $Y$ sur $Y+$ $a Z+b X^{\prime}$, avec $a, b \in \mathbf{R}$, l'algèbre d'isotropie $\mathcal{I}$ est $\mathbf{R} Y$. Pour cela, il suffit de montrer que $\mathcal{I}$ n'est pas incluse dans $\mathbf{R} X^{\prime} \oplus \mathbf{R} Z$.

Remarquons que $a d\left(\alpha X^{\prime}+\beta Z\right)(\mathcal{H}) \subset \mathbf{R} X^{\prime} \oplus \mathbf{R} Z, \forall \alpha, \beta \in \mathbf{R}$. Si $\mathcal{I}$ est $\mathbf{R}\left(\alpha X^{\prime}+\beta Z\right)$, alors l'action de $\operatorname{ad}\left(\alpha X^{\prime}+\beta Z\right)$ sur $T_{x_{0}} F \simeq \mathcal{H} / \mathcal{I}$ est donnée par une matrice de rang 1 . Ceci implique que $I$ n'est pas semi-simple. Nous venons de prouver que dans le cas où $I$ est semi-simple, alors $\mathcal{I}$ n'est pas incluse dans $\mathbf{R} X^{\prime} \oplus \mathbf{R} Z$ et on peut donc considérer que $\mathcal{I}=\mathbf{R} Y$.

Démontrons la même chose dans le cas où $I$ est unipotent. Observons d'abord que $\mathcal{I} \neq$ $\mathbf{R} X^{\prime}$, car l'élément central $X^{\prime}$ agit trivialement sur $\mathcal{H}$ et donc aussi sur $\mathcal{H} / \mathcal{I} \simeq T_{x_{0}} F$.

Supposons par l'absurde que $\mathcal{I} \subset \mathbf{R} X^{\prime} \oplus \mathbf{R} Z$. Quitte à appliquer un automorphisme de $\mathcal{H}$ qui envoie $Z$ sur $Z+\alpha X^{\prime}$, avec $\alpha \in \mathbf{R}$, supposons que $\mathcal{I}=\mathbf{R} Z$. Il s'ensuit que l'algèbre commutative $\mathbf{R} X^{\prime} \oplus \mathbf{R} Y$ intersecte trivialement $\mathcal{I}$ et agit donc librement et transitivement sur $F$. Comme dans la preuve de la proposition 6.1, ceci implique que la connexion induite sur $F$ par $\nabla$ est plate et que $H$ est le groupe de Heisenberg. Ceci est absurde : le groupe de Heisenberg et $\mathbf{R} \times A G$ ne sont pas isomorphes.

Il en découle que, modulo un automorphisme de $\mathcal{H}$, on a $\mathcal{I}=\mathbf{R} Y$. Mais ceci est impossible dans le cas unipotent. En effet, de nouveau $\mathbf{R} X^{\prime} \oplus \mathbf{R} Z$ agit librement et transitivement sur $F$ et $F$ est plate. Comme avant, $H$ est isomorphe au groupe de Heisenberg, ce qui contredit notre hypothèse.

Nous venons de démontrer que $H=\mathbf{R} \times A G$ implique que l'isotropie est semi-simple et que $\mathcal{I}$ est engendrée par $Y$. 
Comme l'isotropie $a d(Y)$ fixe le vecteur $X^{\prime}$ et dilate exponentiellement la direction engendrée par $Z$, on peut choisir pour $T$ la deuxième direction isotrope du plan lorentzien $X^{\prime \perp}$. Alors $\operatorname{ad}(Y) \cdot T=[Y, T]=-T+\alpha Y$, pour une certaine constante réelle $\alpha$. Quitte à changer $T$ en $T-\alpha Y$, on suppose que $[Y, T]=-T$.

On montre que $[T, Z]=a X^{\prime}+b Y$, avec $a, b \in \mathbf{R}$ et $\left[T, X^{\prime}\right]=c T$.

La relation de Jacobi donne $[Y,[T, Z]]=[[Y, T], Z]+[T,[Y, Z]]=[-T, Z]+[T, Z]=$ 0 . Ceci montre que $[T, Z]$ centralise $Y$ et doit donc appartenir à l'algèbre engendrée par $Y$ et $X^{\prime}$.

Par ailleurs, comme $X^{\prime}$ et $Y$ commutent, $T$ est également un vecteur propre de $\operatorname{ad}\left(X^{\prime}\right)$ (non seulement de $a d(Y)$ ). Ceci donne $\left[T, X^{\prime}\right]=c T$, avec $c \in \mathbf{R}$.

Considérons maintenant $\mathcal{L}=[\mathcal{G}, \mathcal{G}]$. Comme $[Y, Z]=Z,[Y, T]=-T$ et $[T, Z]=$ $a X^{\prime}+b Y$, nous avons que $\mathcal{L}$ contient l'algèbre de Lie engendrée par $Z, T$ et $a X^{\prime}+b Y$. On remarque que $\left[a X^{\prime}+b Y, Z\right]=b Z$. Ceci implique $b=0$, car sinon l'algèbre de Lie engendrée par les vecteurs $a X^{\prime}+b Y$ et $Z$ est l'algèbre du groupe affine qui ne peut pas être contenue dans l'algèbre nilpotente $\mathcal{L}$.

Nous avons donc $b=0$ et $[T, Z]=a X^{\prime}$. Comme $\left[T, a X^{\prime}\right]=a c T$, le même argument implique que ou bien $a=0$, ou bien $c=0$.

Une renormalisation évidente permet de voir que $a \neq 0$ peut être remplacer par $a=1$ et $c \neq 0$ peut être remplacer $\operatorname{par} c=1$.

Finalement on a trois algèbres de Lie possibles pour $\mathcal{G}$.

1. Si $a=0$ et $c=0$, les relations sont les suivantes : $[Y, Z]=Z,[Y, T]=-T,[T, Z]=0$ et $X^{\prime}$ est central. Le groupe $G$ est alors $\mathbf{R} \times S O L$, où $X^{\prime}$ engendre le centre du groupe et l'algèbre de Lie de sol est engendrée par $Y, Z, T$. Le groupe $S O L$ peut être vu comme un produit semi-directe $\mathbf{R} \ltimes \mathbf{R}^{2}$, où l'action infinitésimale de $Y$ est donnée dans la base $Z, T$, par la matrice $\left(\begin{array}{cc}1 & 0 \\ 0 & -1\end{array}\right)$. Le sous-groupe à un paramètre $Y$ engendre l'isotropie qui se trouve entièrement incluse dans $S O L$.

2. Pour $a=1$ et $c=0$ on trouve $[Y, Z]=Z,[Y, T]=-T,[T, Z]=X^{\prime},\left[T, X^{\prime}\right]=0$ et le groupe correspondant est $\mathbf{R} \ltimes H$ eis.

Le premier facteur $\mathbf{R}$ est engendré par le groupe d'isotropie $Y$. L'action du sous-groupe à un paramètre engendré par le premier facteur sur l'algèbre de Lie de Heisenberg est $\left(X^{\prime}, Z, T\right) \rightarrow\left(X^{\prime}, e^{t} Z, e^{-t} T\right)$. L'élément $X^{\prime}$ qui engendre le centre de Heisenberg est donc également dans le centre de $G$.

Le deuxième facteur, isomorphe au groupe de Heisenberg, intersecte trivialement le groupe d'isotropie et agit donc transitivement $\operatorname{sur} G / I$.

3. Pour $a=0$ et $c=1$ on trouve : $[Y, Z]=Z,[Y, T]=-T,[T, Z]=0,\left[T, X^{\prime}\right]=T$ et on a $G=\mathbf{R}^{2} \ltimes \mathbf{R}^{2}$. L'action adjointe du premier facteur, engendré par $Y$ et $X^{\prime}$ agit sur la copie de $\mathbf{R}^{2}$ engendrée par les éléments $Z$ et $T$ par les matrices $\left(\begin{array}{cc}1 & 0 \\ 0 & -1\end{array}\right)$ et respectivement $\left(\begin{array}{cc}0 & 0 \\ 0 & -1\end{array}\right)$.

Ceci achève la preuve.

6.2 Cas : H isomorphe à Heisenberg

Proposition 6.3 i) L'isotropie I est unipotente.

ii) Les feuilles de $\mathcal{F}$ sont plates (pour la connexion de Levi-Civita) et X est parallèle le long de $\mathcal{F}$. 
Démonstration L'action de $I$ sur $\mathcal{H} / \mathcal{I} \simeq T_{x_{0}} F$ ne préserve aucune décomposition non triviale de $T_{x_{0}} F$. Il en résulte que $I$ est unipotent. Aussi, $I$ est différent du centre de Heisenberg car celui-ci agit trivialement sur $\mathcal{H} / \mathcal{I}$. Ceci implique qu'il existe des copies de $\mathbf{R}^{2}$ transverse à $I$ dans $H$ et qui agissent donc librement et transitivement sur $F$. Comme dans la preuve de la proposition 6.1, ceci implique que $F$ est plate et que $X$ est parallèle en restriction à $F$.

Proposition $6.4 \mathcal{H}$ est un idéal de $\mathcal{G}$.

Corollaire 6.5 Le feuilletage engendré par l'action locale de $\mathcal{H}$ cö̈ncide avec $\mathcal{F}$.

Démonstration Considérons $A$ un champ de Killing et $B$ un champ de vecteurs tangent à $X^{\perp}$. Nous montrons que $[A, B]=\nabla_{A} B-\nabla_{B} A$ est encore dans $X^{\perp}$. Le terme $\nabla_{A} B$ est dans $X^{\perp}$. En effet, $g(B, X)=0$ implique $g\left(\nabla_{A} B, X\right)=-g\left(\nabla_{A} X, B\right)=0$ (d'après la proposition 3.4, $\nabla_{A} X$ est colinéaire à $X$ ).

Par ailleurs, le champ de Killing $A$ préserve $X$ et donc $\nabla_{X} A=\nabla_{A} X$. Comme $\nabla$. A est antisymétrique, on a $g\left(\nabla_{B} A, X\right)=-g\left(B, \nabla_{X} A\right)=-g\left(B, \nabla_{A} X\right)=0$, car $\nabla_{A} X$ est colinéaire à $X$. Le terme $\nabla_{B} A$ est donc également dans $X^{\perp}$, ce qui implique que $[A, B] \in X^{\perp}$.

Structure algèbrique de $\boldsymbol{G}$ De ce qui précède résulte que $G$ est une extension de $H$. Considérons, comme avant, une base $\left\{X^{\prime}, Y, Z\right\}$ de $\mathcal{H}$ telle que $X^{\prime}$ est central, $[Y, Z]=X^{\prime}$ et $Y$ engendre $\mathcal{I}$. Vu l'expression de l'action de $\mathcal{I}=\mathbf{R} Y$ sur $\mathcal{G} / \mathcal{I} \simeq T_{x_{0}} M$, dans une base adaptée, on peut choisir le quatrième générateur $T$ de $\mathcal{G}$ tel que $\operatorname{ad}(Y) T=-Z$ dans $\mathcal{G} / \mathcal{I}$. Il s'ensuit que $[Y, T]=-Z+\beta Y$, avec $\beta \in \mathbf{R}$.

Comme l'action adjointe de $T$ sur $\mathcal{H}$ préserve le centre $\mathbf{R} X^{\prime}$ de $\mathcal{H}$, il existe une constante réelle $c$ telle que $\left[T, X^{\prime}\right]=c X^{\prime}$.

Proposition $6.6 \quad$ i) Il existe une fonction $H$-invariante $f$ sur $G / I$ telle que $X^{\prime}=f X$ (la fonction $f$ est localement définie sur $M$ et constante sur les feuilles de $\mathcal{F}$ ).

ii) $X$ est de Killing (et $f$ est constante) si et seulement si $c=0$.

iii) Dans la base $\left\{X^{\prime}, Z, Y\right\}$ de $\mathcal{H}$ l'action de T est donnée parad $(T)=\left(\begin{array}{ccc}c & m & 0 \\ 0 & c+\beta & 1 \\ 0 & n & -\beta\end{array}\right)$, avec $m, n \in \mathbf{R}$.

iv) Si c $=0$ et $n+\beta^{2}=0$, alors g est une métrique plate, invariante à gauche sur Heis.

Démonstration i) Le champ de droites $\mathbf{R} X^{\prime}$ est $\mathcal{G}$-invariant. Or, dans le cas d'isotropie unipotente, le seul champ de droites $\mathcal{G}$-invariant dans $T M$ est celui engendré par $X$. Donc, $X^{\prime}$ et $X$ sont en tout point colinéaires.

ii) Comme $X$ est $\mathcal{G}$-invariant, il est de Killing si et seulement s'il engendre le centre de $\mathcal{G}$. Ainsi $X$ est de Killing si et seulement s'il est un multiple constant de $X^{\prime}$. Il est équivalent de dire que la fonction $f$ est constante ou que $c=0$.

iii) On applique la relation de Jacobi aux éléments $Y, Z$ et $T$ de $\mathcal{G}$ et on constate que $\operatorname{ad}(T)$ agit comme une dérivation sur $\mathcal{G}$ si et seulement si $a d(T) Z=m X^{\prime}+(c+\beta) Z+n Y$, avec $m, n \in \mathbf{R}$.

iv) Si $c=0$ et $n+\beta^{2}=0$, alors les éléments $X^{\prime}, Z-\beta Y$ et $T$ engendrent une sous-algèbre de Lie de $\mathcal{G}$ isomorphe à l'algèbre de Heisenberg (avec $\mathbf{R} X^{\prime}$ comme centre) qui agit librement et transitivement sur $M$. Il en découle que $g$ est localement isométrique à une métrique invariante à gauche sur Heisenberg qui attribue la norme 0 au générateur du centre. Or, ces métriques lorentziennes sont plates [35].

Remarquons que le feuilletage $\mathcal{F}$ est défini par la forme différentielle $\mathcal{G}$-invariante $g(\cdot, X)$. Ceci implique que $\mathcal{F}$ admet une structure transverse de translation, autrement dit, par chaque 
point passe une transversale locale paramètrée par un ouvert de $\mathbf{R}$ de manière que les holonomies locales agissent par translations. Ce type de structure a été étudiée, entre autres, par Ehresmann, Thurston et Fédida et est amplement décrite dans le livre de Molino [28].

Dans ce cas on a une application développante $\operatorname{dev}: \widetilde{M} \rightarrow \mathbf{R}$ de la structure transverse du feuilletage. L'application dev est une submersion équivariante sous l'action du groupe fondamental de $M$ qui agit sur $\widetilde{M}$ par transformations de revêtement et sur $\mathbf{R}$ via un morphisme d'holonomie (transverse) $\pi_{1}(M) \rightarrow \mathbf{R}$. Ici le groupe d'holonomie transverse s'obtient en considérant la projection sur $G / H \simeq \mathbf{R}$ de l'holonomie de la $(G, G / I)$-géométrie.

Comme $M$ est compacte, l'application développante est complète, i.e. dev est une submersion surjective sur $\mathbf{R}$ et les fibres de $d e v$ sont les feuilles du feuilletage image réciproque de $\mathcal{F}$ par le revêtement universel. De plus, $\widetilde{M}$ est un produit, difféomorphe à $\mathbf{R} \times \widetilde{F}$, où $\widetilde{F}$ est le revêtement universel d'une feuille $F$ de $\mathcal{F}[12,28]$.

\section{Classification : cas de l'isotropie unipotente}

Ici, l'isotropie est supposée unipotente. D'après les propositions 6.1 et 6.2 , nous sommes dans le cas où $H$ est le groupe de Heisenberg. Rappelons que des constantes $c$ et $n$ ont été definies à la proposition 6.6. Le but de cette section est de démontrer la

Proposition 7.1 Soit $(M, g)$ une variété lorentzienne compacte localement modelée sur une géométrie lorentzienne (non nécessairement maximale) $G / I$, avec $G$ résoluble de dimension 4 et I sous-groupe à un paramètre unipotent.

i) Si $c=0$, alors g est localement isométrique à une métrique plate, invariante à gauche sur Heis.

ii) Si $c \neq 0$, alors $M$ est localement modelée sur la géométrie Lorentz-SOL. Les seules réalisations compactes de la géométrie Lorentz-SOL sont, à revêtement fini près, des quotients de SOL par un réseau $\Gamma$.

Démontrons d'abord la complétude via les deux propositions suivantes :

Proposition 7.2 La $(H, H / I)$-structure est complète sur chaque feuille de $\mathcal{F}$. Les feuilles de $\mathcal{F}$ sont homéomorphes à des plans, à des cylindres ou à des tores.

Démonstration Démontrons que la $(H, H / I)$-structure sur une feuille $F$ est complète.

Désignons par $\widetilde{F}$ le revêtement universel de $F$ et par $\widetilde{X}$ l'image réciproque du champ $X$ sur $\widetilde{F}$. L'application développante de notre $(H, H / I)$-structure envoie $\widetilde{F}$ sur un ouvert de $\mathbf{R}^{2}$ et le champ de Killing $\widetilde{X}$ sur le champ $\frac{\partial}{\partial x}$ dans l'espace des paramètres $(h, x)$ de $\mathbf{R}^{2}$. La restriction de $g$ à $F$ est une forme quadratique de rang 1 dont le noyau est engendré par $X$ et qui s'exprime dans le modèle sous la forme $d h^{2}$ : elle confère au feuilletage engendré par $X$ une structure transverse de translation.

Le champ $\widetilde{X}$ étant complet (car $X$ est défini sur la variété compacte $M$ ), pour chaque orbite de $\widetilde{X}$ l'application développante réalise un difféomorphisme entre un ouvert connexe de $\widetilde{F}$ contenant l'orbite en question et invariant par $\widetilde{X}$ et une bande ouverte horizontale de la forme $] h-\epsilon, h+\epsilon\left[\times \mathbf{R}\right.$ dans $\mathbf{R}^{2}$.

Il reste à démontrer que la structure transverse de translation est complète (nous ne pouvons pas utiliser directement le résulat de [12,28] car $F$ n'est pas nécessairement compacte).

On construit un champ de vecteurs $V \in X^{\perp}$, globalement défini sur $M$, et de norme constante égale à 1 . Pour cela il suffit de choisir un champ de vecteurs dans $X^{\perp}$ qui n'est en aucun point colinéaire à $X$ (à revêtement double près, il n'y a pas de contrainte topologique pour 
cela) et de le diviser par sa norme (cette norme est non nulle car le noyau de la restriction de $g$ à $F$ est engendré par $X$ ). La construction de $V$ est purement topologique et n'utilise pas l'homogénéité locale.

Comme les orbites de $V$ sont paramétrées par la longueur, le flot de $V$ envoie une orbite de $X$ sur une autre orbite de $X$ (sans respecter nécessairement le paramétrage). L'image réciproque $\widetilde{V}$ de $V$ par le revêtement universel de $F$ est un champ de vecteurs complet sur $\widetilde{F}$ (car $V$ complet sur $M$ ). L'image de $\widetilde{V}$ par l'application développante de la $(H, H / I)$ géométrie est un champ de vecteurs complet sur $\mathbf{R}^{2}$ de $d h^{2}$-norme constante égale à 1 . Ceci montre que l'application développante est surjective sur $\mathbf{R}^{2}$ et que la structure de translation transverse du feuilletage engendré par $X$ est complète sur $F$. On conclut à la complétude la $(H, H / I)$-géométrie de $F$.

Passons à présent au type topologique des feuilles de $\mathcal{F}$. Comme les feuilles admettent le champ de vecteurs non singulier $X$, si elles sont compactes elles sont nécessairement homéomorphes à des tores.

Il reste à traiter le cas d'une feuille $F$ non compacte. Son groupe fondamental $\pi_{1}(F)$ agit sur le revêtement universel $\mathbf{R}^{2}$ en préservant les flots des champs de vecteurs $\widetilde{X}$ et $\widetilde{V}$. Il s'ensuit que $\pi_{1}(F)$ agit sur la transversale du feuilletage engendré par $\widetilde{X}$ en préservant le flot de $\widetilde{V}$ : ceci donne un morphisme de $\pi_{1}(F)$ dans $\mathbf{R}$. Le noyau de ce morphisme est formé par les éléments de $\pi_{1}(F)$ qui fixent les feuilles données par l'action de $\widetilde{X}$ : ce sous-groupe de $\pi_{1}(F)$ commute avec le flot de $\widetilde{X}$ et est donc également commutatif. Il en découle que $\pi_{1}(F)$ ne peut être un groupe libre, sans être isomorphe à $\mathbf{Z}$ ou trivial. Dans ce cas $F$ est nécessairement homéomorphe à un cylindre ou à un plan.

Proposition 7.3 La $(G, G / I)$-structure de M est complète.

Démonstration Comme les feuilles de $\mathcal{F}$ sont complètes par la proposition 7.2 et que le feuilletage $\mathcal{F}$ admet une structure transverse de translation, l'application développante sera un difféomorphisme dès que la structure transverse de translation est complète. Or, on a vu que la compacité de $M$ implique la complétude de la structure transverse [28] (ou [12], théorème 1.10).

\section{Lemme 7.4 $\quad$ i) Le groupe d'holonomie $\Gamma$ n'est pas contenu dans $H$.}

ii) $\Gamma$ n'est pas contenu dans un sous-groupe abélien connexe de $G$.

iii) $S i \Gamma$ est abélien, alors $c \neq 0$ et $\Gamma$ intersecte non trivialement $H$.

iv) Si $c=0$ et $\Gamma$ est nilpotent, alors g est plate, invariante à gauche sur Heis.

Démonstration Grâce à la proposition 7.3, l'application développante est un difféomorphisme et $M$ s'identifie au quotient de $G / I$ par le groupe d'holonomie $\Gamma$.

i) Supposons par l'absurde que $\Gamma \subset H$. Rappelons que $H$ est le sous-groupe distingué de $G$ qui fixe chaque feuille du feuilletage $\mathcal{F}$ de $G / I$. L'application développante descend alors en une application continue de $M$ sur le quotient de $H \backslash G / I$. Or, ce quotient, qui s'identifie à la transversale du feuilletage $\mathcal{F}$ est homéomorphe à $\mathbf{R}$ et ne peut être l'image continue du compact $M:$ absurde.

ii) Supposons par l'absurde que $\Gamma$ est contenu dans un sous-groupe de Lie abélien connexe de dimension deux $\bar{\Gamma}$ de $G$. Un calcul immédiat montre que le centralisateur dans $\mathcal{H}$ d'un élément appartenant à la différence $\mathcal{G} \backslash \mathcal{H}$ est de dimension égale à 1 , engendré par $X^{\prime}$, si $c=0$, ou par un élément de $\mathcal{H}$ non contenu dans $\mathbf{R} X^{\prime} \oplus \mathbf{R} Y$, si $c \neq 0$. Il en découle que $\bar{\Gamma}$ est de dimension au plus deux.

Traitons d'abord le cas où $\bar{\Gamma}$ est un sous-groupe à un paramètre (non contenu dans $H$ ). Dans ce cas le double quotient $\bar{\Gamma} \backslash G / I$ s'identifie à $H / I$ qui n'est pas compact. 
L'application développante descend en une application continue de $M$ sur $\bar{\Gamma} \backslash G / I$. Comme $M$ est compacte, l'image devrait être compacte : contradiction.

Traitons maintenant le cas où $\bar{\Gamma}$ est de dimension 2 .

Si $c \neq 0$, alors $\bar{\Gamma}$ intersecte $H$ selon un sous-groupe à un paramètre engendré par un élément $Z^{\prime}$ de $\mathcal{H}$ non contenu dans $\mathbf{R} X^{\prime} \oplus \mathbf{R} Y$. Le groupe $\bar{\Gamma}$ est engendré alors par le sous-groupe à un paramètre associé à $Z^{\prime}$ et par n'importe quel sous-groupe à un paramètre $l$, contenu dans $\bar{\Gamma}$ et transverse à $H$. Le quotient $\bar{\Gamma} \backslash G / I$ s'identifie alors au quotient à gauche de $H / I$ par le sous-groupe à un paramètre engendré par $Z^{\prime}$.

Comme $Z^{\prime} \notin \mathbf{R} X^{\prime} \oplus \mathbf{R} Y$, l'algèbre commutative $\mathbf{R} X^{\prime} \oplus \mathbf{R} Z^{\prime}$ agit transitivement sur les feuilles de $\mathcal{F}$ : dans les coordonnées affines des feuilles, l'action se fait par translations. En particulier, le champ de Killing $Z^{\prime}$ est parallèle, et en restriction à la feuille $H / I$ de $\mathcal{F}$, une orbite du champ de vecteurs complet $X$ constitue une transversale totale au feuilletage défini par $Z^{\prime}$. Ceci implique que le quotient de $H / I \simeq \mathbf{R}^{2}$ par l'action de $Z^{\prime}$ s'identifie à la transversale $\mathbf{R}$ du feuilletage trivial définit par $Z^{\prime}$. Or, $\mathbf{R}$ ne peut pas être l'image continue du compact $M:$ absurde.

Si $c=0, \bar{\Gamma}$ est engendré par $X^{\prime}$ et par n'importe quel sous-groupe à un paramètre $l$ contenu dans $\bar{\Gamma}$ et transverse à $H$. Le quotient $\bar{\Gamma} \backslash G / I$ s'identifie alors au quotient à gauche de $H / I$ par le sous-groupe à un paramètre engendré par le centre $X^{\prime}$. Ce quotient est isomorphe à $\mathbf{R}$ qui n'est pas compact : absurde.

iii) Considérons la projection $p: G \rightarrow G / \exp \left(\mathbf{R} X^{\prime}\right)$ sur le quotient de $G$ par le centre de $H$. Observons que $G / \exp \left(\mathbf{R} X^{\prime}\right)$ est isomorphe au produit semi-direct $\mathbf{R} \ltimes \mathbf{R}^{2}$, où le facteur $\mathbf{R}^{2}$ est le quotient de $H$ par son centre et l'action infinitésimale de $\mathbf{R}$ sur $\mathbf{R}^{2}$ s'exprime par la matrice $A=\left(\begin{array}{cc}c+\beta & 1 \\ n & -\beta\end{array}\right)$ (voir la proposition 6.6).

Remarquons que dans le cas où $A$ engendre un groupe à un paramètre de rotations de $\mathbf{R}^{2}$, le produit semi-direct correspondant $\mathbf{R} \ltimes \mathbf{R}^{2}$ est isomorphe au revêtement universel du groupe des déplacements du plan euclidien et admet un centre non trivial isomorphe à $\mathbf{Z}$ (sous-groupe discret formé par les rotations d'angle un multiple entier de $2 \pi$ ).

Traitons d'abord le cas où $A$ n'engendre pas un groupe à un paramètre de rotations de $\mathbf{R}^{2}$. Dans ce cas, le centralisateur d'un élément qui n'est pas une translation pure dans $\mathbf{R} \ltimes \mathbf{R}^{2}$ est un groupe de Lie abélien connexe de dimension 1 ou 2, selon que le noyau de la matrice précédente est de dimension 0 ou 1 respectivement.

Soit $\Gamma_{1}$ l'image de $\Gamma$ par $p$. Alors $\Gamma_{1}$ est abélien et contient au moins un élément qui n'est pas une translation pure : $\Gamma$ n'est pas contenu dans $H$ (par le point i). Il en découle que $\Gamma_{1}$ est contenu dans un sous-groupe de Lie abélien connexe de dimension deux $L$ de $G / \exp \left(\mathbf{R} X^{\prime}\right)$. Remarquons que $p^{-1}(L)$ est un sous-groupe connexe de dimension deux ou trois qui n'est pas contenu dans $H$.

Dans le cas $c=0, X^{\prime}$ engendre le centre de $G$ et $p^{-1}(L)$ est un sous-groupe abélien connexe de $G$ contenant $\Gamma$ : impossible par le point ii.

Dans le cas $c \neq 0, p^{-1}(L)$ est isomorphe au groupe affine $A G$ ou au produit $A G \times \mathbf{R}$. Dans les deux cas le centralisateur d'un élément non central est un groupe de Lie abélien connexe (sous-groupe à un paramètre dans le cas de $A G$ et de dimension deux dans le cas $A G \times \mathbf{R})$. Il s'ensuit que $\Gamma$ est contenu dans un groupe de Lie abélien connexe : impossible par ii.

Supposons maintenant que $A$ engendre un groupe à un paramètre de rotations.

Si $c=0$, la trace de $A$ est nulle et, par conséquent, $A$ est une rotation d'angle $\frac{\pi}{2}$. L'action correspondante de $\mathbf{R}$ sur $\mathbf{R}^{2}$ transite donc par le groupe cyclique à 4 
éléments et, quitte à considérer un revêtement fini d'ordre 4 de $M$, on peut considérer que l'action de $\mathbf{R}$ sur $\mathbf{R}^{2}$ est triviale : on est ramené au cas précédent.

Nous avons donc démontré que $c \neq 0$. Les sous-groupes abéliens maximaux $L \mathrm{du}$ revêtement universel du groupe des déplacements du plan sont les sous-groupes à un paramètre conjugués au groupe des rotations et le groupe engendré par les translations et le centre $\mathbf{Z}$ du groupe.

Supposons par l'absurde que $\Gamma_{1}$ ne contient pas de translation pure non triviale. Alors $\Gamma_{1}$ est contenu dans un sous-groupe à un paramètre et on conclut, comme avant, que $\Gamma$ est contenu dans un sous-groupe abélien connexe de $G$ : absurde.

Il reste que $\Gamma_{1}$ contient au moins une translation pure non triviale et, par conséquent, $\Gamma$ contient au moins un élément non trivial de $H$.

iv) Comme $G$ n'est pas nilpotent, il s'ensuit que $\bar{\Gamma}$ est de dimension 3, isomorphe au groupe de Heisenberg. On a donc que l'algèbre de Lie dérivée de l'algèbre de Lie de $\bar{\Gamma}$ est $\mathbf{R} X^{\prime}$. Or, si $T+a Y+b Z$ et $T+a^{\prime} Y+b^{\prime} Z$ sont deux éléments linéairement indépendants du quotient de l'algèbre de Lie de $\bar{\Gamma}$ par $\mathbf{R} X^{\prime}$ (avec $a, b$ et $a^{\prime}, b^{\prime}$ des constantes réelles), leur crochet qui vaut $\left(a-a^{\prime}\right)(Z-\beta Y)+\left(b-b^{\prime}\right)(n Y+\beta Z)$ n'est nul que si le déterminant $n+\beta^{2}$ s'annule. Donc $n+\beta^{2}=0$ et la proposition 6.6 implique alors que $g$ est plate.

Passons maintenant à la preuve de la proposition 7.1 :

Démonstration (i) Supposons d'abord $c=0$. D'après la proposition 6.6, $X$ est alors un champ de Killing isotrope globalement défini.

Il est prouvé dans [41] (voir section 14 : le cas $a=0$, qui correspond à notre géométrie $G / I$ avec $c=0$ ) que le champ de Killing $X$ est nécessairement équicontinu. Par définition, ceci signifie que le flot de $X$ préserve également une métrique riemannienne sur $M$. Nous pouvons donc utiliser la classification des champs de Killing riemanniens sur les variétés compactes de dimension 3 [6]. Comme $X$ préserve aussi bien une métrique lorentzienne, le feuilletage engendré par $X$ est transversalement de translation, modelé (transversalement) sur $\mathbf{R}^{2}$ agissant par translations sur lui-même [2]. Un résultat de [28] (chapitre 4, théorème 4.2) affirme alors que les adhérences des orbites de $X$ ont toutes la même dimension. Selon la dimension de l'adhérence des orbites de $X$ les situations possibles sont les suivantes [6] (voir également le théorème 4.2 de [2]):

1) Si les orbites de $X$ sont denses dans $M$, alors $M$ s'identifie à un tore $T^{3}$ sur lequel le flot de $X$ est à orbites denses et le feuilletage engendré par $X$ est linéaire. Comme le groupe fondamental de $T^{3}$ est abélien, il en découle que $\Gamma$ est abélien, ce qui est impossible par le lemme 7.4.

2) Si les adhérences des orbites sont de dimension 2, il est montré également dans [6] (voir le théorème 1 de la section III.A et le corollaire 4 de la section III.B) que $M$ est un tore $T^{3}$ de dimension 3. On conclut comme précédemment.

3) Il reste à traiter le cas où les orbites de $X$ sont fermées. Dans ce cas, à revêtement fini près, $M$ est une fibration principale sur un tore $T^{2}$ ou bien sur une sphère $S^{2}$ avec le champ de Killing $X$ qui engendre la fibration principale [6] (le cas des fibrés de Seifert non triviaux est éliminé grâce à l'existence de la structure transverse de translation : l'holonomie d'une orbite de $X$ ne peut pas être une rotation rationnelle non triviale car elle ne doit pas avoir de point fixe). 
Comme les orbites de $X$ sont fermées, l'holonomie $\Gamma$ intersecte non trivialement le centre de $H$ et cette intersection est un sous-groupe discret isomorphe à $\mathbf{Z}$ (image du groupe fondamental de la fibre par le morphisme d'holonomie).

Ainsi l'holonomie $\Gamma$ est une extension centrale d'un groupe abélien (l'image du groupe fondamental de $T^{2}$ ) et est donc nilpotente. Le lemme 7.4 implique alors que $g$ est plate, invariante à gauche sur Heis.

ii) Considérons maintenant le cas $c \neq 0$.

Prouvons d'abord que $\Gamma \cap H$ est un sous-groupe $\Delta$ non trivial de $\Gamma$.

Supposons par l'absurde que $\Gamma \cap H=\{1\}$. Il s'ensuit donc que $\Gamma$ s'injecte dans $G / H \simeq \mathbf{R}$, ce qui implique que $\Gamma$ est commutatif, et contredit le lemme 7.4.

Comme les feuilles de $\mathcal{F}$ sont complètes, une telle feuille s'identifie au quotient de $H / I$ par l'action (à gauche) de $\Delta$. Il en découle que $\Delta$ s'identifie au groupe fondamental d'une feuille de $\mathcal{F}$ et est un sous-groupe discret de $H$ isomorphe à $\mathbf{Z}$ (les feuilles sont homéomorphes à des cylindres) ou à $\mathbf{Z} \oplus \mathbf{Z}$ (les feuilles sont homéomorphes à des tores).

Considérons $\gamma$ un élément de $\Gamma$ ayant une projection non-triviale sur $G / H$. Pour ce qui va suivre, on ne fera plus appel aux crochets exacts de $T$ avec $Y$ et $Z$. On se permettra donc de modifier $T$ en lui ajoutant un élément de $\mathcal{H}$. On peut ainsi supposer que $\gamma=\exp (\alpha T)$, pour un certain $\alpha \in \mathbf{R}$. Quitte à changer $T \operatorname{par}-T$ et $\gamma \operatorname{par} \gamma^{-1}$, on suppose que $\alpha>0$ et $c<0$.

Considérons maintenant l'action de $\gamma$ sur $H$, et en particulier sur $\Delta$ et sur sa fermeture de Zariski $\bar{\Delta}$ dans $H$. Notons que $H$ étant un groupe algébrique réel, $\bar{\Delta}$ est bien définie. Les groupes algébriques admettant un nombre fini de composantes connexes, on peut supposer, quitte à considérer un revêtement fini de $M$, que $\bar{\Delta}$ est connexe.

Considérons d'abord le cas $\Delta \simeq \mathbf{Z}$. Comme $\mathbf{Z}$ n'admet pas d'automorphismes autres que $z \rightarrow-z$, à indice 2 près, $\Gamma$ agit trivialement sur $\Delta$, et donc également sur le groupe à un paramètre $\bar{\Delta}$. Comme $c \neq 0$, l'élément $\gamma$ ne préserve pas $X^{\prime}$ et donc $\bar{\Delta}$ admet un générateur infinitésimal $Z^{\prime} \in \mathcal{H}$, non colinéaire à $X^{\prime}$.

Il s'ensuit aussi que $\Gamma$ est contenu dans le centralisateur $C$ de $\bar{\Delta}$ dans $G$. Le sousgroupe $C$ de $G$ admet un centre de dimension au moins 1 et est donc de dimension au plus 3 (comme $c \neq 0$, le centre de $G$ est trivial).

L'algèbre de Lie $\mathcal{C}$ de $C$ contient $\mathbf{R} X^{\prime} \oplus \mathbf{R} Z^{\prime}$, et doit également contenir $T$. Ainsi $\mathcal{C}$ est définie par les relations $\left[T, Z^{\prime}\right]=\left[X^{\prime}, Z^{\prime}\right]=0$ et $\left[T, X^{\prime}\right]=c X^{\prime}$. Comme $\Delta$ et (donc) $\bar{\Delta}$ agissent proprement sur $H / I$, il s'en suit que $\mathbf{R} Z^{\prime}$ intersecte trivialement l'isotropie $\mathbf{R} Y$. Il en découle que le groupe $C$ agit librement et transitivement sur le modèle $G / I$ et que $M$ admet une $(C, C)$-structure. En particulier, $\Gamma$ est un réseau (cocompact) de $C$ et $M$ s'identifie au quotient $\Gamma \backslash C$. Or, $C$ est isomorphe à $\mathbf{R} \times A G$ et n'admet pas de réseau car il n'est pas unimodulaire. Ceci est absurde.

Considérons à présent le cas $\Delta \simeq \mathbf{Z}^{2}$. Alors $\bar{\Delta}$ est abélien et de dimension 2 . Il doit contenir le centre de $H$. Notons son algèbre de Lie $\mathbf{R} X^{\prime} \oplus \mathbf{R} Z^{\prime}$, où $Z^{\prime}$ est un élément de $\mathcal{H}$ non colinéaire à $X^{\prime}$.

Comme les feuilles de $\mathcal{F}$ sont des tores et que le feuilletage admet une structure de translation transverse, un résultat bien connu [28] (chapitre 3, proposition 3.7) assure que $M$ est un fibré en tores sur un cercle et l'image de $\Gamma$ par la projection $G \rightarrow G / H$ (l'holonomie de la structure de translation transverse de $\mathcal{F}$ ) est un sous-groupe discret isomorphe à $\mathbf{Z}$. On peut alors supposer que l'élément choisi $\gamma$ engendre la projection de $\Gamma$ sur $G / H$. Il s'ensuit alors que $\Gamma$ est inclus dans le groupe engendré par $\gamma$ et $\Delta$. 
Rappelons que $\gamma$ agit sur le plan $\bar{\Delta}$ en préservant le réseau $\Delta$. Mais le groupe préservant un réseau est unimodulaire. Il en découle que $\gamma$ agit sur $\bar{\Delta}$ en préservant le volume. Comme $\left[T, X^{\prime}\right]=c X^{\prime}$, l'action de $\gamma$ admet une valeur propre égale à $e^{\alpha c}<1$. Il s'ensuit que l'action de $\gamma$ sur $\mathbf{R} X^{\prime} \oplus \mathbf{R} Z^{\prime}$ est diagonalisable, avec deux valeurs propres $e^{\alpha} c$ et $e^{-\alpha c}$. Ainsi, l'algèbre de Lie engendrée par $X^{\prime}, Z^{\prime}$ et $T$ est isomorphe à sol. Comme avant, $\mathbf{R} X^{\prime} \oplus \mathbf{R} Z^{\prime}$ intersecte trivialement l'isotropie et $S O L$ agit librement et transitivement sur $G / I$. Par la proposition 1.2, nous sommes en présence d'une géométrie Lorentz-SOL, car l'algèbre dérivée engendrée par $X^{\prime}$ et $Z^{\prime}$ est dégénérée et la direction propre $\mathbf{R} X^{\prime}$ de $a d(T)$ est isotrope. Par ailleurs, nous avons montré que l'holonomie $\Gamma$ est contenue dans $S O L$. Il en découle que $M$ possède une ( $S O L, S O L)$-structure, ce qui implique que $M$ est un quotient de $S O L$ par un réseau cocompact.

\section{Classification: cas de l'isotropie semi-simple}

L'isotropie est supposée semi-simple. Traitons d'abord le cas

\section{$8.1 G$ résoluble}

Le but de cette partie est de démontrer la

Proposition 8.1 Soit $(M, g)$ une variété lorentzienne compacte localement modelée sur une géométrie lorentzienne (non nécessairement maximale) $G / I$, avec $G$ résoluble de dimension 4 et I sous-groupe à un paramètre semi-simple. Alors g est localement isométrique, ou bien à une métrique plate, invariante à gauche sur SOL, ou bien à la métrique Lorentz-Heisenberg.

Les seules réalisations compactes de la géométrie Lorentz-Heisenberg sont, à revêtement fini près, des quotients de Heis par un réseau $\Gamma$.

On utilise la classification des algèbres $\mathcal{G}$ obtenue dans la proposition 6.2.

Cas $G=\mathbf{R} \times S O L$.

Proposition 8.2 La géométrie $(G, G / I)$ représente une métrique plate invariante à gauche sur SOL. Elle n'est donc pas maximale.

Démonstration Dans ce cas $G=\mathbf{R} \times S O L$, où $S O L$ est engendré par $\{Z, T, Y\}$ (avec les relations $[Y, Z]=Z,[Y, T]=-T$ et $[T, Z]=0$ ) et le centre est engendré par $X^{\prime}$. L'algèbre de Lie engendrée par $\left\{X^{\prime}, Z, T\right\}$ est abélienne et agit librement transitivement sur $G / I$. La métrique $g$ est donc plate. Cette métrique s'identifie à une métrique lorentzienne invariante à gauche sur le groupe $S O L$ engendré par les éléments $\left\{Y+X^{\prime}, Z, T\right\}$.

Le modèle $G / I$ ne représente pas une géométrie lorentzienne maximale : la géométrie maximale correspondante est la géométrie Minkowski.

Cas $G=\mathbf{R} \ltimes$ Heis.

Proposition 8.3 La géométrie $(G, G / I)$ est la géométrie Lorentz-Heisenberg.

Démonstration Le centre de $G$ est non trivial et engendré par un élément central $X^{\prime}$ de Heis. Ceci donne l'existence d'un champ de Killing globalement défini sur $M$ de norme 
constante positive et qui est préservé par l'action de $\mathcal{G}$. Il coïncide donc avec un multiple du champ de Killing $X$ stabilisé par l'isotropie.

Le deuxième facteur $H$ eis agit librement et transitivement sur $G / I$. Ceci implique que la métrique $g$ s'identifie localement à une métrique invariante à gauche sur Heis qui attribue à l'élément central $X^{\prime}$ une norme strictement positive. La variété $M$ est alors localement modelée sur la géométrie Lorentz-Heisenberg (voir Sect. 4).

Démontrons maintenant la complétude et la rigidité de Bieberbach des réalisations compactes de la géométrie Lorentz-Heisenberg.

Démonstration Dans le cas où le champ de Killing $X$ (de norme égale à 1$)$ est non-équicontinu, il a été montré dans [41] (théorème 0) que $X$ est nécessairement un flot d'Anosov dont les feuilletages stables et instables sont les deux droites isotropes de $X^{\perp}$. Comme $\mathcal{G}$ est résoluble, $M$ est nécessairement une suspension d'un difféomorphisme hyperbolique d'un tore et $g$ est plate (voir [41], preuve du Théorème 2). Ceci est absurde car les seules métriques lorentziennes plates et invariantes par translations sur $\mathrm{Heis}$ attribuent à l'élément central $X^{\prime}$ la norme 0 [35].

Il reste à analyser le cas où le flot de $X$ est équicontinu. Le feuilletage engendré par $X$ admet une structure transverse qui est à la fois lorentzienne et riemannienne. En particulier, le feuilletage engendré par $X$ est transversalement de translation (modelé sur $\mathbf{R}^{2}$ agissant sur lui-même) et la dimension de l'adhérence d'une orbite de $X$ ne dépend pas de l'orbite choisie [28] (chapitre 4, théorème 4.2). Selon la valeur de cette dimension nous avons les possibilités suivantes [6] (voir également le théorème 4.2 dans [2]):

1) Si les orbites de $X$ sont denses dans $M$ alors $M$ s'identifie à un tore $T^{3}$ sur lequel le flot de $X$ est à orbites denses et le feuilletage engendré par $X$ est linéaire. Précisons qu'il est classiquement connu que le groupe des isométries riemanniennes lisses est fermé dans le groupe des homéomorphismes [31] (voir aussi [23], théorème 3.10). L'adhérence du flot de $X$ dans le groupe des homémorphismes de $M$ est alors un groupe de Lie abélien compact qui agit transitivement par isométries, aussi bien pour la métrique lorentzienne $g$, que pour une métrique riemannienne. Ceci implique que l'adhérence du flot de $X$ est un tore $T^{3}$ qui agit simplement transitivement et par isométries sur $M$. Il en résulte que $g$ est également plate : absurde.

2) Si les adhérences des orbites de $X$ sont de dimension 2, il est montré dans [6] (voir le théorème 1 de la section III.A et le corollaire 4 de la section III.B) que $M$ est un tore $T^{3}$. Par ailleurs, l'adhérence du flot de $X$ dans les homéomorphismes de $M$ est un tore $T^{2}$ qui agit par isométries pour la métrique lorentzienne $g$, ce qui entraîne l'existence de deux champs de Killing périodiques et qui commutent sur $M$. Ceci implique que le groupe d'holonomie $\Gamma$ contient un sous-groupe discret isomorphe à $\mathbf{Z}^{2}$.

Comme le groupe fondamental de $T^{3}$ est abélien, il s'ensuit que l'holonomie $\Gamma$ est également abélienne.

Considérons l'adhérence de Zariski $\bar{\Gamma}$ de $\Gamma$ dans $G$ (notons qu'ici $G$ est isomorphe en tant que groupe de Lie à un groupe algébrique réel). Comme $\Gamma$ est abélien et contient un sous-groupe discret isomorphe à $\mathbf{Z}^{2}$, son adhérence de Zariski $\bar{\Gamma}$ est un sous-groupe de Lie abélien de dimension au moins 2 de $G$. Quitte à considérer un revêtement fini de $M, \bar{\Gamma}$ sera supposé connexe.

Supposons par l'absurde que $\Gamma$ n'est pas contenu dans Heis.

On constate que le centralisateur dans l'algèbre de Lie heis d'un élément de $\mathcal{G}$ qui ne se trouve pas dans heis est exactement $\mathbf{R} X^{\prime}$. Donc $\bar{\Gamma}$ est de dimension 2. Plus précisément, $\bar{\Gamma}$ est une copie de $\mathbf{R}^{2} \subset G$ engendrée par $\mathbf{R} X^{\prime}$ et par n'importe quel sous-groupe à un paramètre $l$ contenu dans $\mathbf{R}^{2}$ et transverse à $\mathbf{R} X^{\prime}$. 
L'image de l'application développante est un ouvert $U$ de $G / I$. Nous avons donc une application surjective de $M$ dans le quotient de $U$ par l'action de $\bar{\Gamma}$. Comme le champ de Killing $X$ est globalement défini sur $M$, l'ouvert $U$ est invariant par l'action de $X^{\prime}$.

Pour comprendre le quotient à gauche de $G / I$ par $\bar{\Gamma}$, il est aisé de regarder d'abord le quotient de $G$ par son centre, engendré par $\mathbf{R} X^{\prime}$. On constate que ce quotient est isomorphe au groupe $S O L$ et que le quotient à gauche de $G / I$ par $\bar{\Gamma}$ s'identifie au quotient à gauche de $S O L / I$ par l'image $l^{\prime}$ de $l$ dans $G / I \simeq S O L$. Le modèle $S O L / I$ est le plan de Minkowski et l'image de l'ouvert saturé $U$ dans $S O L / I$ est un ouvert $U^{\prime}$.

L'application développante fournit alors une application surjective de $M$ dans le quotient de $U^{\prime}$ par le champ de Killing $l^{\prime} \subset S O L$, non contenu dans les translations (car $l$ non contenu dans Heis).

La contradiction recherchée viendra du fait que la norme de $l^{\prime}$ (constante sur les orbites de $l^{\prime}$ ) descend en une fonction continue sur $M$ sans extremas locaux.

En effet, pour préciser cette idée considérons des coordonnées $(z, t)$ sur $S O L / I$ dans lesquelles la métrique lorentzienne s'exprime $q=d z d t$. Comme $l^{\prime}$ est un champ de Killing qui n'est pas une translation pure, alors quitte à le multiplier par une constante, $l^{\prime}=\left(z \frac{\partial}{\partial z}-t \frac{\partial}{\partial t}\right)+a \frac{\partial}{\partial z}+b \frac{\partial}{\partial t}$, avec $a, b \in \mathbf{R}$. Dans ce cas l'expression de $q\left(l^{\prime}\right)$ est la fonction $(z+a)(b-t)$ qui n'admet pas d'extrema local sur $\mathbf{R}^{2}$.

Pourtant la fonction $q\left(l^{\prime}\right)$ descend bien en une fonction continue non constante sur la variété compacte $M$ : elle devrait donc admettre sur l'ouvert $U^{\prime}$ au moins un minimum et un maximum. Cette contradiction achève la preuve de $\Gamma \subset \mathrm{Heis}$ (à indice fini près), dans le cas où l'adhérence d'une orbite de $X$ est de dimension 2 . Comme l'holonomie $\Gamma$ est incluse dans $H$ eis qui agit librement et transitivement sur $G / I$, notre ( $G, G / I$ )-géométrie sur $M$ se réalise alors comme une (Heis, Heis)-géométrie. La (Heis, Heis)-géométrie étant de type riemannien, elle est complète et $M$ s'identifie (à revêtement fini près) à un quotient de Heis par un réseau cocompact.

3) Il reste à régler le cas où toutes les orbites de $X$ sont fermées, de dimension 1. Dans ce cas $M$ est une fibration principale en cercles sur un tore $T^{2}$, la fibration principale étant engendrée par $X$ (voir théorème 4.2 dans [2]). Les orbites de $X$ étant fermées, le groupe d'holonomie $\Gamma$ intersecte le sous-groupe à un paramètre engendré par l'élément central $X^{\prime}$ selon un sous-groupe discret isomorphe à $\mathbf{Z}$. Il en découle que le sous-groupe à un paramètre engendré par $X^{\prime}$ est contenu dans $\bar{\Gamma} \cap \mathrm{Heis}$, où $\bar{\Gamma}$ est l'adhérence de Zariski de $\Gamma$.

Il en résulte que l'holonomie $\Gamma$ est une extension centrale d'un groupe abélien (l'image du groupe fondamental de $T^{2}$ par le morphisme d'holonomie). Le groupe $\Gamma$ est donc nilpotent.

Supposons par l'absurde que $\Gamma$ n'est pas contenu dans Heis. Alors l'adhérence de Zariski $\bar{\Gamma}$ de $\Gamma$ est un sous-groupe nilpotent de $G$ qui contient le centre $\mathbf{R} X^{\prime}$ et qui n'est pas contenu dans $G$. Comme avant, on peut supposer $\bar{\Gamma}$ connexe.

On montre que $\bar{\Gamma}$ est nécessairement de dimension 2. D'abord, la dimension ne peut être 4 car $G$ n'est pas nilpotent.

Le centralisateur dans l'algèbre de Lie heis d'un élément de $\mathcal{G}$ qui ne se trouve pas dans heis est exactement $\mathbf{R} X^{\prime}$. Par ailleurs, si l'algèbre de Lie de $\bar{\Gamma}$ est supposée de dimension 3, alors l'intersection de l'algèbre de Lie de $\bar{\Gamma}$ avec heis est de dimension 2. Or, le crochet de Lie d'un élément appartenant à la différence $\mathcal{G} \backslash$ hei s avec un élément de hei $\backslash \mathbf{R} X^{\prime}$ n' est jamais contenu dans $\mathbf{R} X^{\prime}$. Notre algèbre ne peut donc pas être nilpotente et de dimension 3. 
Il s'ensuit que $\bar{\Gamma}$ est une copie de $\mathbf{R}^{2} \subset G$ engendrée par $\mathbf{R} X^{\prime}$ et par n'importe quel sous-groupe à un paramètre $l$ contenu dans $\mathbf{R}^{2}$ et transverse à $\mathbf{R} X^{\prime}$. On conclut alors comme dans le cas précédent. Plus précisément, le quotient de l'image de l'application développante par $\bar{\Gamma}$ s'identifie au quotient d'un ouvert de $S O L / I$ par le feuilletage trivial engendré par $X^{\prime}$. Or, ce quotient est séparé et non compact et ne peut être l'image continue de $M$ : absurde.

Nous venons de démontrer que (à indice fini près) $\Gamma \subset$ Heis. On conclut comme dans le cas précédent que (à revêtement fini près) $M$ est un quotient de Heis par un réseau cocompact.

\section{$8.2 G$ non resoluble}

Il reste à régler le cas de la géométrie produit apparue au point (2) de la proposition 5.1. Dans ce cas, $G=\mathbf{R} \times S \widetilde{L(2, \mathbf{R})}$ et $I \subset S \widetilde{L(2, \mathbf{R})}$ est un groupe à un paramètre semi-simple.

Proposition 8.4 Il n'existe pas de réalisation compacte de $(G, G / I)$.

Démonstration Il s'agit d'un cas simple d'application de la méthode développée dans [1] (voir également la section 2.2.2 du rapport de survol [24]) dont nous présentons brièvement le principe.

La projection naturelle $G \rightarrow G / I$ munit $G / I$ d'un fibré en droites réelles $G$-invariant, qui est donc bien défini sur $M$. La forme linéaire sur $\mathcal{G}$, qui s'annule sur le centre $\mathbf{R}$ et qui coïncide sur $\operatorname{sl}(2, \mathbf{R})$ avec la forme duale (par rapport à la forme de Killing) d'un générateur de $\mathcal{I}$, équipe ce fibré d'une connexion $G$-invariante dont la forme de courbure $\omega$ est une forme volume en restriction aux orbites de $S L(2, \mathbf{R})$. La 2-forme $\omega$ est alors bien définie sur $M$, où elle s'annule sur $X$ et est non dégénérée en restriction aux feuilles de $X^{\perp}$.

Par ailleurs, le fibré en droites précédent admettant une section jamais nulle (eventuellement sur un revêtement double de $M$ ), la forme de courbure $\omega$ est exacte, égale à la différentielle de la 1-forme de connexion $\omega_{1}$ associée à une section.

Considérons à présent la 1-forme différentielle $\mu$ sur $M$ qui s'annule sur $X^{\perp}$ et telle que $\mu(X)=1$ (il s'agit de la forme duale de $X$ par rapport à $g$ ). Comme le flot de $X$ préserve le champ de plans intégrable $X^{\perp}$, la 1 -forme $\mu$ est fermée.

La forme différentielle $\omega \wedge \mu$ est une forme volume sur $M$. Par ailleurs, cette forme est exacte, égale à $d\left(\omega_{1} \wedge \mu\right)$. Si $M$ est compacte, ceci est impossible.

\section{References}

1. Benoist, Y., Labourie, F.: Sur les espaces homogènes modèles de variétés compactes. Publ. Math. I.H.E.S. 76, 99-109 (1992)

2. Boubel, C., Mounoud, P., Tarquini, C.: Lorentzian foliations on 3-manifolds. Ergod. Th. Dynam. Sys. 26, 1339-1362 (2006)

3. Bromberg, S., Medina, A.: Geodesically complete Lorentzian metrics on some homogeneous 3 manifolds. SIGMA 4, 13 paper 88 (2008)

4. Calabi, E., Markus, L.: Relativistic space forms. Ann. of Math. 75, 63-76 (1962)

5. Calvaruso, G.: Homogeneous structures on three-dimensional Lorentzian manifolds. J. Geom. Phys. 57, 1279-1291 (2007)

6. Carrière, Y.: Flots riemanniens, dans Structures transverses des feuilletages. Toulouse, Astérisque 116, 3152 (1984)

7. Carrière, Y.: Autour de la conjecture de L. Markus sur les variétés affines. Invent. Math. 95, 615-628 (1989)

8. Cordero, L., Parker, P.: Left-invariant Lorentzian metrics on 3-dimensional Lie groups. Rendiconti di Matematica VII(17), 129-155 Roma (1997) 
9. D’Ambra, G.: Isometry groups of Lorentz manifolds. Invent. Math. 92, 555-565 (1988)

10. D'Ambra, G., Gromov, M.: Lectures on transformations groups: geometry and dynamics. Surveys in Differential Geometry, pp. 19-111, Cambridge (1990)

11. Dumitrescu, S.: Dynamique du pseudo-groupe des isométries locales sur une variété lorentzienne analytique de dimension 3. Ergod. Th. Dynam. Sys. 28(4), 1091-1116 (2008)

12. Epstein, D.: Transversaly hyperbolic 1-dimensional foliations, dans Structures transverses des feuilletages. Toulouse, Astérisque 116, 53-68 (1984)

13. Fried, D., Goldman, W.: Three-dimensional affine crystallographic groups. Adv. Math. 47(1), 1-49 (1983)

14. Goldman, W.: Nonstandard Lorentz space forms. J. Diff. Geom. 21(2), 301-308 (1985)

15. Goldman, W., Kamishima, Y.: The fundamental group of a compact flat Lorentz space form is virtually polycyclic. J. Diff. Geom. 19(1), 233-240 (1984)

16. Gromov, M.: Rigid transformation groups, Géométrie Différentielle. In: Bernard et Choquet-Bruhat, D. (ed.) Travaux en cours, vol. 33, pp. 65-141. Hermann, Paris (1988)

17. Guediri, M.: Sur la complétude des pseudo-métriques invariantes à gauche sur les groupes de Lie nilpotents. Rend. Sem. Mat. Univ. Politec. Torino 52(4), 371-376 (1994)

18. Guediri, M., Lafontaine, J.: Sur la complétude des variétés pseudo-riemanniennes. J. Geom. Phys. 15(2), 150-158 (1995)

19. Guediri, M.: On completeness of left-invariant Lorentz metrics on solvable Lie groups. Rev. Mat. Univ. Complut. Madrid 9(2), 337-350 (1996)

20. Kirilov, A.: Eléments de la théorie des représentations. M.I.R., Moscou (1974)

21. Kowalski, O.: Counter-example to the second Singer's theorem. Ann. Global Anal. Geom. 8(2), 211214 (1990)

22. Klingler, B.: Complétude des variétés Lorentziennes à courbure sectionnelle constante. Math. Ann. 306, 353-370 (1996)

23. Kobayashi, S., Nomizu, T.: Foundations of Differential Geometry I. Interscience Publishers, London (1963)

24. Labourie, F.: Quelques résultats récents sur les espaces localement homogènes compacts, Symposia Mathematica (en l'honneur d'Eugenio Calabi), pp. 267-283 (1996)

25. Lastaria, F., Tricceri, F.: Curvature-orbits and locally homogeneous Riemannian manifolds. Ann. Mat. Pura Appl. 165(4), 121-131 (1993)

26. Mess, G.: Lorentz spacetimes of constant curvature, preprint IHES/M/90/28 (1990)

27. Milnor, J.: Curvatures of left invariant metrics on lie groups. Adv. Math. 21, 293-329 (1976)

28. Molino, P.: Riemannian Foliations, Birkhauser, Boston, Basel (1988)

29. Morrill, M.: Nonexistence of compact de Sitter manifolds, PHD, University of California (1996)

30. Mostow, G.: The extensibility of local lie groups of transformations and groups on surfaces. Ann. Math. 52(2), 606-636 (1950)

31. Myers, S., Steenrod, N.: The group of isometry of a Riemannian manifold. Ann. Math. 40, 400-416 (1939)

32. Nomizu, K.: Left-invariant Lorentz metrics on Lie groups. Osaka J. Math. 16, 143-150 (1979)

33. Patrangenaru, V.: Locally homogeneous pseudo-Riemannian manifolds. J. Geom. Phys. 17, 59-72 (1995)

34. Rahmani, S.: Métriques de Lorentz sur les groupes de Lie unimodulaires de dimension 3. J. Geom. Phys. 9, 295-302 (1992)

35. Rahmani, N., Rahmani, S.: Lorentzian geometry of the Heisenberg group. Geom. Dedicata 118, 133140 (2006)

36. Salein, F.: Variétés anti-de Sitter de dimension 3 exotiques. Ann. Inst. Fourier, Grenoble 50(1), 257284 (2000)

37. Scott, P.: The Geometries of 3-manifolds. Bull. London Math. Soc. 15, 401-487 (1983)

38. Thurston, W.: Three dimensional manifolds, Kleinian groups and hyperbolic geometry. Bull. Am. Math. Soc. 6(3), 357-381 (1982)

39. Thurston, W.: The geometry and topology of 3-manifolds. Princeton University Press, Princeton (1983)

40. Wolf, J.: Spaces of constant curvature. McGraw-Hill Series in Higher Math (1967)

41. Zeghib, A.: Killing fields in compact Lorentz 3-manifolds. J. Diff. Geom. 43, 859-894 (1996)

42. Zeghib, A.: Remarks on Lorentz symmetric spaces. Compositio Math. 140(6), 1675-1678 (2004) 